PFC/JA-96-32

\title{
ENHANCED COUPLING OF THE FAST WAVE TO ELECTRONS THROUGH MODE CONVERSION TO THE ION HYBRID WAVE
}

\author{
C. N. Lashmore-Davies, V. Fuchs, \\ A. K. Ram, and A. Bers
}

September 1996

\author{
Plasma Fusion Center \\ Massachusetts Institute of Technology \\ Cambridge, Massachusetts 02139 USA
}

This work was supported in part by U.K. Department of Trade and Industry and Euratom; by Atomic Energy of Canada Ltd, HydroQuebec, and Institut National de la Recherche Scientifique; and by the U.S. Department of Energy Contract No. DE-FG02-91ER-54109. Reproduction, translation, publication, use and disposal, in whole or part, by or for the United States Government is permitted.

To be published in Physics of Plasmas. 


\section{ENHANCED COUPLING OF THE FAST WAVE TO ELECTRONS THROUGH MODE CONVERSION TO THE ION HYBRID WAVE}

C. N. Lashmore-Davies, V. Fuchs,

A. K. Ram, and A. Bers

TABLE OF CONTENTS

Abstract . . . . . . . . . . . . . . . . . . . 1

1. Introduction . . . . . . . . . . . . . . . . . 2

2. The Reduced Dispersion Relation . . . . . . . . . . . . . . . . . . . 4

3. Ion Bernstein Waves in a Two-Ion Species Plasma . . . . . . . . . . . . . . . 6

4. Mode Conversion of the Fast Wave to the Ion Hybrid Wave . . . . . . . . . . . 8

5. The Damping of the Fast Wave . . . . . . . . . . . . . . . . . . . . . . 11

6. The Damping of the Ion Hybrid Wave . . . . . . . . . . . . . . . . . . . . 13

7. Mode Conversion and Direct Damping . . . . . . . . . . . . . 16

8. Summary and Conclusions . . . . . . . . . . . . . . 18

9. Acknowledgements . . . . . . . . . . . . . . . . . . . . . . . . . . . . . 19

Appendix A . . . . . . . . . . . . . . . . 20

References . . . . . . . . . . . . . . . . . . 22

Figures . . . . . . . . . . . . . . . . . . . 23 


\title{
Enhanced Coupling of the Fast Wave to Electrons through Mode Conversion to the Ion Hybrid Wave
}

\author{
C N Lashmore-Davies ${ }^{1}$, V Fuchs ${ }^{2}, \mathrm{~A} \mathrm{~K} \mathrm{Ram}{ }^{3}$ and A Bers ${ }^{3}$ \\ ${ }^{1}$ UKAEA, Fusion, Culham, Abingdon, Oxon, OX14 3DB, UK \\ (UKAEA/Euratom Fusion Association) \\ ${ }^{2}$ Centre canadien de fusion magnétique, 1804 Montée Ste Julie, Varennes, Québec, \\ J3X 1S1, Canada \\ ${ }^{3}$ Plasma Fusion Center, MIT, Cambridge, MA 02139, USA
}

\begin{abstract}
The mode conversion of the fast compressional Alfvén wave to the ion hybrid wave is analyzed with particular reference to a plasma with two ion species present in approximately equal proportions. Two configurations are considered, the first referring to the usual resonance-cut-off case and the second to a cut-off-resonance-cut-off situation. The optimum conditions for maximising the mode converted energy are given. The second order fast wave equation is generalised to include the effect of the parallel electric field. Hence, all ion and electron loss mechanisms for the fast wave are incorporated, including mode conversion at the two-ion hybrid resonance. The significance of the approximate equality of the two ion species concentrations is that the mode converted ion hybrid wave is damped only by the electrons. The damping of the ion hybrid wave is described with the aid of the local dispersion relation and by means of a toroidal ray tracing code. In particular, the ray tracing calculation shows that the mode converted energy is totally absorbed by the electrons close to the two-ion hybrid resonance. The generalised fast wave equation is solved to determine how much energy is lost from the fast wave, incident from the low field side, before it enounters the two-ion hybrid resonance. For comparable concentrations of the two ion species, the mode converted power can be separated from the power directly absorbed by the ions and electrons from the fast wave. This allows the conditions to be ascertained under which strong electron heating through mode conversion dominates the direct dissipation of the fast wave.
\end{abstract}




\section{INTRODUCTION}

Ion cyclotron heating is used on many present generation magnetic fusion experiments and is one of the auxiliary heating methods under consideration for ITER. Fast waves in the ion cyclotron range of frequencies can interact with both electrons and ions. Ion heating occurs in the vicinity of the ion cyclotron resonances whereas electron heating requires substantial values of the electron beta. For current drive applications it is of interest to choose conditions such that the incident fast wave damps predominantly

on the electrons. Such conditions can be achieved either by operating below all ion cyclotron frequencies or by working at a higher frequency such that only the weakly absorbing high ion cyclotron harmonics (say three and above) are present in the plasma. While ITER will achieve these high electron betas, present experiments do not reach conditions for which the fast wave undergoes strong single pass absorption on the electrons.

In this paper we analyse another possibility. This scheme is based on the mode conversion which can occur when the two-ion hybrid resonance exists in the plasma. Since the fast wave antenna is situated on the low field side of present large tokamaks, the occurrence of the ion hybrid resonance normally leads to strong reflection of the fast wave from the associated hybrid cut-off. This is particularly true for $\mathrm{D}(\mathrm{H})$ plasmas. When the hybrid resonance is well separated from the minority (hydrogen) resonance, strong reflection occurs and very little mode conversion is produced. If $k_{\|}$is increased or the minority concentration is reduced so that the minority and hybrid resonances overlap, reflection is reduced, strong minority ion cyclotron damping occurs and again there is very little mode conversion. For this reason Ram et $a l^{1}$ concentrated on small hydrogen concentrations and small values of the parallel wavenumber $k_{\|}$in their discussion of current drive by mode converted ion Bernstein waves.

The fraction of fast wave energy incident from the low field side which can be mode converted depends on the distance between the hybrid cut-off and resonance positions. Jacquinot et $a l^{2}$ drew attention some time ago to the sensitivity of this distance to $k_{\|}$. Recently, Majeski et al ${ }^{3}$ have again emphasised this point by noting that significant mode conversion can occur in D-T, D- ${ }^{3} \mathrm{He}$ plasmas of equal ion concentrations and high values of the fast wave parallel wave number, $k_{\|}$. In addition, Majeski et al ${ }^{3}$ have also drawn attention to the effect that the fast wave cut-off on the high-field side has on the mode-conversion which occurs in the vicinity of the two-ion hybrid resonance. Two configurations must be considered. The first is the well known one of a cut-off-resonance first treated by Budden ${ }^{4}$, which is relevant when the cut-off on the high-field side is not present in the plasma. The second refers to the case of three critical points, arising when the high-field side cut-off is present in the plasma (the usual situation, in fact). This cut-off-resonance-cut-off triplet was emphasised by Majeski et al and solved in the two-ion hybrid context by Fuchs et al ${ }^{5}$. For the Budden case, the condition for fifty per cent transmission corresponds to the 
maximum fraction of twenty five per cent mode converted energy in a single transit. In the case of the triplet the mode converted fraction can be one hundred per cent ${ }^{5}$ for a single transit, provided there is no other damping. This condition depends on the species mix and the value of the parallel wave number.

For a plasma with two ion species present in comparable proportions the two-ion hybrid resonance is well separated from the regions of ion cyclotron resonance. Hence, the mode converted wave is undamped by the ions in the region of the hybrid resonance. More importantly, we shall show that the mode converted wave is damped strongly by the electrons close to the ion hybrid resonance. Thus, for conditions corresponding to efficient mode conversion, strong localised electron heating can be expected. Furthermore, since the localisation of the heating is governed by the position of the two-ion hybrid resonance, both on- and off-axis heating can be produced. These features have already been observed on TFTR ${ }^{3}$.

The plan of the paper is as follows. Section II contains a general discussion of the dispersion relation for the fast wave in a hot plasma. It is shown that for a cold plasma the fast wave couples to the shear Alfvén wave at the two-ion hybrid resonance, whereas in a hot plasma the fast wave couples to the ion hybrid wave (a member of the family of ion Bernstein waves) when the plasma beta exceeds the electron to ion mass ratio. Section III provides a discussion of the properties of ion Bernstein waves in a plasma containing two ion species. Particular attention is focused on the ion hybrid wave. Section IV is concerned with the behaviour of the fast wave at the two-ion hybrid resonance, and hence mode conversion to the ion hybrid wave. The Budden equation is obtained for a two-ion species plasma relevant to the important case of comparable concentrations and oblique propagation. The cut-off-resonancecut-off triplet, which occurs when the ion densities and the parallel wave number are adjusted so that the fast wave cut-off on the high field side is present in the plasma, is also discussed. In Section $\mathrm{V}$ the fast wave equation is generalized to include the effects of the parallel electric field. Thus, all the ion and electron damping terms are included. Hence this equation is used to calculate the energy lost by the fast wave as it approaches the two-ion hybrid resonance from the low field side. In Section VI the damping of the mode converted ion hybrid wave by the electrons is discussed, first by means of the local dispersion relation and then with the aid of a toroidal ray tracing code. A quantitative analysis of the damping of the fast wave obtained from integration of the fast wave equation is given in Section VII. A comparison of the power mode converted with the power dissipated outside the hybrid resonance region is also presented. Parameters appropriate to JET and TFTR are chosen. Finally, a summary and conclusions are given in Section VIII. 


\section{THE REDUCED DISPERSION RELATION}

Let us begin our discussion from the full, hot plasma, electromagnetic dispersion relation. The dispersion relation is obtained in the usual way from the linearized set of Maxwell's equations and the Vlasov equation. Formally, we write

$$
\left(n^{2} \underline{\underline{I}}-\underline{n} \underline{n}-\underline{\underline{\epsilon}}\right) \underline{E}=0
$$

where $\underline{\epsilon}(\omega, \underline{k})$ is the hot plasma dielectric tensor calculated assuming that all species of particles have a Maxwellian distribution function. We assume a harmonic time and space dependence of the form $\exp i(\underline{k} . \underline{x}-\omega t)$ where $\underline{k}=\left(k_{\perp}, 0, k_{\|}\right)$. In Eq. (1), $\underline{n}=c \underline{k} / \omega$ and $n^{2}=n_{\perp}^{2}+n_{\|}^{2}$. The dispersion relation is obtained by setting the determinant of Eq. (1) to zero. The dispersion relation can be written

$$
\begin{aligned}
\left(n_{\perp}^{2}-\right. & \left.\epsilon_{z z}\right)\left\{\left(n_{\|}^{2}-\epsilon_{x x}\right)\left(n_{\|}^{2}-\epsilon_{y y}\right)+\epsilon_{x y}^{2}+\left(n_{\|}^{2}-\epsilon_{x x}\right) n_{\perp}^{2}\right\}-\left(n_{\|}^{2}-\epsilon_{y y}\right) n_{\|}^{2} n_{\perp}^{2}-n_{\|}^{2} n_{\perp}^{4} \\
= & \epsilon_{y z}\left\{\epsilon_{x y}\left(n_{\perp} n_{\|}+\epsilon_{x z}\right)-\epsilon_{y z}\left(n_{\|}^{2}-\epsilon_{x x}\right)\right\}+\epsilon_{x z}\left\{\epsilon_{x y} \epsilon_{y z}+\left(n_{\perp} n_{\|}+\epsilon_{x z}\right)\left(n^{2}-\epsilon_{y y}\right)\right\} \\
& +n_{\|} n_{\perp}\left\{\epsilon_{x y} \epsilon_{y z}+\epsilon_{x z}\left(n^{2}-\epsilon_{y y}\right)\right\}
\end{aligned}
$$

In the limit of a cold plasma the right-hand-side of Eq. (2) vanishes. Comparing the order of magnitude of the terms on the right-hand-side with the dominant terms on the left-hand-side, we find that the right-hand-side can be neglected when $\left(v_{T i} n_{\|} / c\right)^{2} \ll 1$, a condition which is well satisfied in present tokamaks. We can simplify Eq. (2) further by taking advantage of the dominance of the $\varepsilon_{z z}$-element in the ion cyclotron range of frequencies. Retaining only those terms proportional to $\varepsilon_{z z}$, we obtain the usual fast wave approximation, which neglects the effect of the parallel electric field. In Section VI we shall generalize the fast wave approximation to include the parallel electric field through the retention of the off-diagonal terms on the right-hand-side of Eq. (2). The final simplification of Eq. (2) is made through the usual finite Larmor radius expansion. We write the elements of the dielectric tensor to first order in $\lambda_{j}=k_{\perp}^{2} v_{T j}^{2} / 2 \Omega_{j}^{2}$, where $v_{T_{j}}^{2}=2 T_{j} / m_{j}$, the subscript $j$ refers to the $j$-th charge species, and $\Omega_{j}$ is the cyclotron frequency of the species. The elements can be obtained from $\mathrm{Stix}^{6}$ and are

$$
\begin{aligned}
\epsilon_{x x} & =S_{\circ}+\frac{1}{2} \sigma_{1} n_{\perp}^{2} \\
\epsilon_{y y} & =S_{\circ}+\frac{1}{2} \sigma_{2} n_{\perp}^{2} \\
\epsilon_{x y} & =i D_{\circ}+\frac{i}{2} \delta n_{\perp}^{2}
\end{aligned}
$$

The quantities $S_{0}$ and $D_{0}$ are given by

$$
S_{o}=1+\sum_{j} \frac{\omega_{p j}^{2}}{2 \omega^{2}} \zeta_{o j}\left[Z\left(\zeta_{1 j}\right)+Z\left(\zeta_{-1 j}\right)\right]
$$




$$
D_{o}=-\frac{\omega_{p e}^{2}}{\omega \Omega_{e}}+\sum_{j} \frac{\omega_{p j}^{2}}{2 \omega^{2}} \zeta_{\circ j}\left[Z\left(\zeta_{1 j}\right)-Z\left(\zeta_{-1 j}\right)\right]
$$

where

$$
\zeta_{n j}=\left(\omega-n \Omega_{j}\right) / k_{\|} v_{T j}
$$

In the above expressions $\Omega_{j}$ and $\Omega_{e}$ are all positive quantities.

$$
\begin{aligned}
& \sigma_{1}= \sum_{j} \frac{\omega_{p j}^{2}}{\omega^{2}} \zeta_{o j} \frac{v_{T j}^{2}}{2 \Omega_{j}^{2}} \frac{\omega^{2}}{c^{2}}\left[-Z\left(\zeta_{1 j}\right)-Z\left(\zeta_{-1 j}\right)+Z\left(\zeta_{2 j}\right)+Z\left(\zeta_{-2 j}\right)\right] \\
& \sigma_{2}= \sum_{j} \frac{\omega_{p j}^{2}}{\omega^{2}} \zeta_{o j} \frac{v_{T j}^{2}}{2 \Omega_{j}^{2}} \frac{\omega^{2}}{c^{2}}\left[-3 Z\left(\zeta_{1 j}\right)-3 Z\left(\zeta_{-1 j}\right)\right. \\
&\left.+4 Z\left(\zeta_{o j}\right)+Z\left(\zeta_{2 j}\right)+Z\left(\zeta_{-2 j}\right)\right]+\frac{\omega_{p e}^{2}}{\omega^{2}} \zeta_{o e} \frac{v_{T e}^{2}}{2 \Omega_{e}^{2}} \frac{\omega^{2}}{c^{2}} 4 Z\left(\zeta_{o e}\right) \\
& \delta=\sum_{j} \frac{\omega_{p j}^{2}}{\omega^{2}} \zeta_{o j} \frac{v_{T j}^{2}}{2 \Omega_{j}^{2}} \frac{\omega^{2}}{c^{2}}\left[-2 Z\left(\zeta_{1 j}\right)+2 Z\left(\zeta_{-1 j}\right)+Z\left(\zeta_{2 j}\right)-Z\left(\zeta_{-2 j}\right)\right]
\end{aligned}
$$

Neglecting the right-hand-side of Eq. (2) and substituting Eqs. (3) - (5) into Eq. (2), a sixth order equation is obtained. This can be reduced to a fourth order equation by the usual approximation of neglecting electron inertia. The dominant terms on the left-hand-side of Eq. (1) are those proportional to $\epsilon_{z z}$. The resulting fourth order equation is

$$
\begin{aligned}
\left(\frac{1}{2} \sigma_{1}+\frac{\delta^{2}}{4}-\frac{\sigma_{1} \sigma_{2}}{4}\right) n_{\perp}^{4}+\left[S_{o}-n_{\|}^{2}\right. & \left.-\frac{1}{2}\left(\sigma_{1}+\sigma_{2}\right)\left(S_{o}-n_{\|}^{2}\right)+\delta D_{o}\right] n_{\perp}^{2} \\
& -\left(S_{o}-D_{o}-n_{\|}^{2}\right)\left(S_{\circ}+D_{o}-n_{\|}^{2}\right)=0
\end{aligned}
$$

This equation is valid provided $\mu_{o} n_{o} T_{i} / B_{o}^{2}>m_{e} / m_{i}$. The significance of Eq. (12) is the following. For a cold plasma the fast Alfvén wave couples to the shear Alfvén wave in the vicinity of the two-ion hybrid resonance, given by $S_{0}=0$. However, for a hot plasma, the fast wave couples to the ion hybrid wave, which is a member of the family of ion Bernstein waves. The dispersion relation given in Eq. (12) will be used as the basis for an approximate analysis of the fast wave in a multi-ion species plasma in the presence of the two-ion hybrid resonance. Before discussing the propagation and absorption of the fast wave, the properties of ion Bernstein waves in a two-ion species plasma will be considered. 


\section{ION BERNSTEIN WAVES IN A TWO-ION SPECIES PLASMA}

In a hot plasma where $\beta_{i}>m_{e} / m_{i}$, the fast wave couples to the ion hybrid wave near to the two-ion hybrid resonance. Since most previous discussions of ion Bernstein waves have been restricted to a single ion species it is worthwhile to consider the important case of a two-ion species plasma. Although we shall be concerned ultimately with oblique propagation of the fast wave, it is helpful to examine the properties of ion Bernstein waves for the special case of perpendicular propagation.

The electrostatic dispersion relation for ion Bernstein waves propagating at right angles to a uniform magnetic field can be written in the form

$$
1-2 \sum_{j} \frac{\omega_{p j}^{2}}{\omega^{2}} \sum_{n=1}^{\infty} \frac{n^{2} e^{-\lambda_{j}}}{\lambda_{j}} I_{n}\left(\lambda_{j}\right) \frac{\omega^{2}}{\left(\omega^{2}-n^{2} \Omega_{j}^{2}\right)}=0
$$

where the summation over $j$ is over all ion species and the electrons. The effect of the electrons can be neglected for long wavelengths $\left(\lambda_{j} \ll 1\right)$ but must be included for short wavelengths $\left(\lambda_{j} \gg 1\right)$. Consider the case of a two-ion species plasma. The dispersion relation can be written in the form

$$
1+\frac{\omega_{p e}^{2}}{\Omega_{e}^{2}}-2 \sum_{n=1}^{\infty}\left[\Lambda_{1 n} \frac{n^{2} \omega_{p 1}^{2}}{\left(\omega^{2}-n^{2} \Omega_{1}^{2}\right)}+\Lambda_{2 n} \frac{n^{2} \omega_{p 2}^{2}}{\left(\omega^{2}-n^{2} \Omega_{2}^{2}\right)}\right]=0
$$

where only the $n=1$ electron term has been included, to zero order in $\lambda_{e}$, since $\lambda_{e} \ll 1$. The subscripts 1 and 2 denote the two ion species and

$$
\Lambda_{j n}=\frac{e^{-\lambda_{j}}}{\lambda_{j}} I_{n}\left(\lambda_{j}\right) \quad ; \quad j=1,2
$$

Combining the two ion terms, the dispersion relation becomes

$$
1+\frac{\omega_{p e}^{2}}{\Omega_{e}^{2}}-2 \sum_{n=1}^{\infty} n^{2}\left(\Lambda_{1 n} \omega_{p 1}^{2}+\Lambda_{2 n} \omega_{p 2}^{2}\right) \frac{\left(\omega^{2}-n^{2} \Omega_{h n}^{2}\right)}{\left(\omega^{2}-n^{2} \Omega_{1}^{2}\right)\left(\omega^{2}-n^{2} \Omega_{2}^{2}\right)}=0
$$

where

$$
\Omega_{h n}^{2}=\frac{\left(\Lambda_{1 n} \omega_{p 1}^{2} \Omega_{2}^{2}+\Lambda_{2 n} \omega_{p 2}^{2} \Omega_{1}^{2}\right)}{\left(\Lambda_{1 n} \omega_{p 1}^{2}+\Lambda_{2 n} \omega_{p 2}^{2}\right)}
$$

For $\lambda_{1} \ll 1, \lambda_{2} \ll 1$ we have $\Lambda_{11} \simeq \frac{1}{2}, \Lambda_{21} \simeq \frac{1}{2}$, giving

$$
\Omega_{h 1}^{2} \simeq \frac{\left(\omega_{p 1}^{2} \Omega_{2}^{2}+\omega_{p 2}^{2} \Omega_{1}^{2}\right)}{\left(\omega_{p 1}^{2}+\omega_{p 2}^{2}\right)}
$$

which will be recognised as the two-ion hybrid resonance frequency for a cold plasma. 
For a single ion species plasma, the lowest frequency ion Bernstein wave in the long wavelength limit $\left(\lambda_{j} \rightarrow 0\right)$ occurs just below the second harmonic cyclotron frequency, $\omega \lesssim 2 \Omega$. However, for two ion species it can be seen, on examination of Eq. (16), that the lowest frequency solution in the long wavelength limit begins just below the two-ion hybrid frequency. No solution is possible in the long wavelength limit for $\Omega_{h 1}<\omega<\Omega_{2}$. In the short wavelength limit, $\lambda_{j} \gg 1, \omega \rightarrow \Omega_{1}$ from above, where we assume that $\Omega_{2}>\Omega_{1}$. We distinguish this wave from other Bernstein waves by referring to it as the ion hybrid wave. The lowest hybrid frequency, $\Omega_{h 1}$, introduces this additional mode because $\Lambda_{j 1}\left(\lambda_{j}\right) \rightarrow \frac{1}{2}$ as $\lambda_{j} \rightarrow 0$, whereas all other $\Lambda_{j n}\left(\lambda_{j}\right) \rightarrow 0$ as $\lambda_{j} \rightarrow 0$. Thus, the ion hybrid wave lies in the frequency range $\Omega_{1}<\omega<\Omega_{h 1}$. No solutions occur between $\Omega_{h 1}$ and $\Omega_{2}$.

The first ion-Bernstein wave, for long wavelengths, begins just below $2 \Omega_{1}$ (provided $2 \Omega_{1} \neq \Omega_{2}$ ) and tends asymptotically to $\Omega_{2}$ from above for short wavelengths. The particular case of a deuterium-tritium plasma with equal concentrations of deuterium and tritium is shown in Fig 1. The first few $\omega$ versus $k_{\perp}$ curves are shown. The lowest branch is the ion hybrid wave. The first ion Bernstein wave starts from its cut-off just below $2 \Omega_{1}$ and tends asymptotically to $\Omega_{2}$ from above. The next ion Bernstein wave begins just below $2 \Omega_{2}$ (which is, of course, degenerate with $3 \Omega_{1}$ ) and tends asymptotically to $2 \Omega_{1}$. The following ion Bernstein wave has its cut-off just below $4 \Omega_{1}$ and ends on $2 \Omega_{2}$ (which is also $3 \Omega_{1}$ ). The last ion Bernstein wave shown starts just below $3 \Omega_{2}$ and tends asymptotically to $4 \Omega_{1}$. The dispersion curves shown in Fig 1 illustrate the crucial difference between the ion hybrid wave and the ion Bernstein waves. The ion hybrid wave is the only one which does not start from a harmonic of one of the ion cyclotron frequencies. Thus, when a finite value of the parallel wave number is included, all the ion Bernstein waves will be subject to ion cyclotron damping for values of $k_{\perp}$ near to the cut-off, which increases in strength as $k_{\|}$ increases. In contrast, the ion hybrid wave (for comparable ion densities) propagates at frequencies between the two ion cyclotron frequencies and is therefore much less susceptible to ion cyclotron damping. The dispersion relation for the ion hybrid wave for oblique propagation is therefore well approximated by the dispersion relation for perpendicular propagation. The significance of this property of the ion hybrid wave is that it is, therefore, much more likely to be excited by mode conversion of the fast Alfvén wave in ion cyclotron heating experiments, even for large values of $k_{\|}$, provided the two ion species are present in comparable proportions.

The electromagnetic dispersion relation for propagation at right angles to the magnetic field is shown in Fig 2, for which the parameters are the same as in Fig 1. The dispersion curves in Fig 2 show the strong coupling between the compressional Alfven wave and the ion hybrid wave and the much weaker coupling at the two ion cyclotron frequencies. This is indicated by the size of the gap between the coupled branches. The cyclotron harmonic waves at the two fundamental cyclotron frequencies are not Bernstein waves but arise from the electromagnetic terms in the dispersion relation. These electromagnetic cyclotron harmonic waves were first analyzed by Akhiezer et $a l^{7}$ and have also been discussed more recently by Chow et al ${ }^{8}$. 


\section{MODE CONVERSION OF THE FAST WAVE TO THE ION HYBRID WAVE}

An explicit calculation of the mode conversion of the fast Alfvén wave into the ion hybrid wave requires the solution of a fourth order differential equation. Such a fourth order equation can be obtained from the (fourth order) dispersion relation given by Eq. (12). In this section we will establish the transmission, reflection and mode conversion coefficients for the fast wave crossing the two-ion hybrid resonance in a plasma with two ion species in arbitrary proportions and for oblique propagation. Since we shall be most interested in the case where the two ion species are present in comparable proportions, ion cyclotron damping will be negligible in the region of the two-ion hybrid resonance. In view of this we can obtain this information from the cold plasma model by solving the Budden equation.

Thus, neglecting the finite Larmor radius corrections in Eq. (12), we obtain the well known fast wave refractive index

$$
n_{\perp}^{2}=\frac{\left(S_{o}-D_{o}-n_{\|}^{2}\right)\left(S_{o}+D_{o}-n_{\|}^{2}\right)}{\left(S_{0}-n_{\|}^{2}\right)}
$$

The cold plasma approximation is now obtained by expanding $S_{0}$ and $D_{0}$ asymptotically to obtain

$$
n_{\perp}^{2}=\frac{\left[\frac{\omega_{p 1}^{2}}{\Omega_{1}\left(\omega+\Omega_{1}\right)}+\frac{\omega_{p 2}^{2}}{\Omega_{2}\left(\omega+\Omega_{2}\right)}-n_{\|}^{2}\right]\left[\frac{\omega_{p 1}^{2}}{\Omega_{1}\left(\omega-\Omega_{1}\right)}+\frac{\omega_{p 2}^{2}}{\Omega_{2}\left(\omega-\Omega_{2}\right)}+n_{\| 1}^{2}\right]}{\left[\frac{\omega_{p 1}^{2}}{\left(\omega^{2}-\Omega_{1}^{2}\right)}+\frac{\omega_{p 2}^{2}}{\left(\omega^{2}-\Omega_{2}^{2}\right)}+n_{11}^{2}\right]}
$$

We expand the dispersion relation about the position of the ion hybrid resonance, where the wave frequency, $\omega$, satisfies the resonance condition

$$
\omega^{2}=\frac{\omega_{p 1}^{2} \Omega_{2}^{2}+\omega_{p 2}^{2} \Omega_{1}^{2}}{\omega_{p 1}^{2}+\omega_{p 2}^{2}} \equiv \Omega_{i i}^{2}
$$

The equilibrium magnetic field is assumed to vary as $B_{0}(x)=B_{0}(R)\left(1-\frac{x}{R}\right)$. After some algebra, Eq. (20) can be written as

$$
\frac{c_{A}^{2} k_{\perp}^{2}}{\Omega_{i i}^{2}} \simeq \frac{k_{o}^{2}\left(\xi-\xi_{c}\right)}{\left(\xi-\xi_{R}\right)}
$$

where

$$
k_{\circ}^{2}=\left(\frac{\Omega_{i i}}{\Omega_{1}}+1\right)\left(\frac{\Omega_{i i}}{\Omega_{2}}+1\right)\left[\frac{\rho_{1}}{\left(\frac{\Omega_{i i}}{\Omega_{1}}+1\right)\left(\rho_{1}+\rho_{2}\right)}+\frac{\rho_{2}}{\left(\frac{\Omega_{i i}}{\Omega_{2}}+1\right)\left(\rho_{1}+\rho_{2}\right)}-N_{\| 1}^{2}\right]
$$




$$
\begin{gathered}
\xi_{c}=-R_{A}-\frac{R_{A}\left(\omega_{p 1}^{2} \Omega_{2}+\omega_{p 2}^{2} \Omega_{1}\right)}{\left(\omega_{p 1}^{2}+\omega_{p 2}^{2}\right)^{1 / 2}\left(\omega_{p 1}^{2} \Omega_{2}^{2}+\omega_{p 2}^{2} \Omega_{1}^{2}\right)^{1 / 2}}-R_{A} N_{\|}^{2}\left(\frac{\Omega_{i i}}{\Omega_{1}}-1\right)\left(\frac{\Omega_{i i}}{\Omega_{2}}-1\right) \\
\xi_{R}=-R_{A} N_{\|}^{2}\left(\frac{\Omega_{i i}^{2}}{\Omega_{1}^{2}}-1\right)\left(\frac{\Omega_{i i}^{2}}{\Omega_{2}^{2}}-1\right)
\end{gathered}
$$

and $\xi=\Omega_{i i} x / c_{A}, R_{A}=R \Omega_{i i} / c_{A}, N_{\|}=c_{A} k_{\|} / \Omega_{i i}$. The Alfvén speed $c_{A}$ for a two ion species plasma with arbitrary densities is given by $c_{A}^{2}=B_{0}^{2} /\left(\mu_{0}\left(\rho_{1}+\rho_{2}\right)\right)$, where $\rho_{1,2}$ are the mass densities of the two ion species. Taking the inverse Fourier transform of Eq. (22), we obtain Budden's equation for a general, two ion species plasma, in the ion hybrid resonance region

$$
\frac{d^{2} E_{y}}{d \xi^{2}}+\frac{k_{0}^{2}\left(\xi-\xi_{c}\right) E_{y}}{\left(\xi-\xi_{R}\right)}=0
$$

The equation for the fast wave, given by Eq. (26), is valid for arbitrary density ratios. However, since the equation has been derived from the cold model it is particularly relevant to the case when the two ion species are present in approximately equal proportions. This is because, as already mentioned, the effect of ion cyclotron damping is minimized under these circumstances. The relevance of the Budden model to this case is that it enables the proportion of energy mode converted to the ion hybrid wave to be calculated. This energy is then dissipated as the ion hybrid wave propagates away from the hybrid resonance region towards the fundamental resonance of the heavier (lower frequency) ion species. Since mode conversion can occur for significant values of $k_{\|}$and since the ion hybrid wave is a slow, short wavelength mode, it is expected that the wave energy will be dissipated predominantly by the electrons before the wave enters the region of strong ion cyclotron damping.

In order to calculate the mode converted energy, we must compute the transmission coefficient for the fast wave crossing the two-ion hybrid resonance. This is immediately obtained from Eq. (26) and is given by ${ }^{4}$

$$
T=\exp \left[-\pi\left|\xi_{c}-\xi_{R}\right| k_{0}\right]
$$

It is well known that the proportion of energy reflected from the cut-off associated with the resonance for a wave incident from the low magnetic field side is given by $R=(1-T)^{2}$ and, therefore, that the fraction of mode converted energy is $M=$ $T(1-T)$. Hence, according to the Budden model, the maximum fraction of the energy incident from the low field side which can be mode converted in a single transit of the resonance is twenty five per cent. This occurs when fifty per cent of the incident energy is transmitted across the resonance. The optimum conditions for a given species mix can be obtained from $\mathrm{Eq}(27)$.

The dependence of the optimum conditions on the ion species, concentrations and parallel wave number was noted by Jacquinot et al ${ }^{2}$. However, Majeski et $a l^{3}$ have 
recently drawn attention to the fact that the full wave treatment of the two-ion hybrid resonance and cut-off region should also be extended to include the effect of the fast wave cut-off on the high field side. This configuration of three critical points, as opposed to the two treated in the Budden equation, is referred to as a triplet. As $k_{\|}$ is varied the fast wave cut-off moves relative to the hybrid resonance and its associated cut-off, which also move relative to each other (but at a much slower rate). Majeski et al originally suggested that optimum mode conversion occurred as the three critical points coalesced. This is not the case, but the triplet configuration means that the energy transmitted across the hybrid resonance is reflected back to the resonance region. Hence, an enhancement of the mode converted energy is possible.

The triplet configuration should be used whenever the high field side fast wave cut-off is present in the plasma, which is usually the case. If the cut-off is not present in the plasma, then the Budden configuration should be used. Even in this case, the transmitted energy can be returned to the resonance region by reflection from the wall. For this situation the single pass result can be enhanced by multiple-passes of the resonance region, but a quantitative assessment requires an explicit calculation of the wall reflection from both the low and high field side of the resonance ${ }^{9}$. For this more involved calculation another key ingredient would be the strength of the damping outside the two-ion hybrid resonance region.

The triplet configuration for a two-ion species plasma has recently been solved by Fuchs et al ${ }^{5}$. An interesting feature of the solutions (both analytic and numerical) is that the Budden transmission coefficient again appears in the analysis. In fact, the condition for maximum mode conversion in the triplet configuration again corresponds to a value of 0.5 for the Budden transmission coefficient and, in addition, to a phase condition. Because of the second cut-off on the high field side, there is no transmitted energy for the triplet case. The incident energy is either reflected or mode converted. Depending on the separation of the high-field side cut-off and the resonance, either the mode conversion is enhanced and the reflection reduced, or vice versa. This separation is a function of the parallel wave number. Hence, the mode conversion and reflection coefficients exhibit oscillatory behaviour as a function of the parallel wave number. For a case with no other losses, the optimum condition gives rise to one hundred per cent mode conversion for a single interaction of the incident wave with the resonance. A similar result was previously obtained by Ngan and Swanson ${ }^{10}$, who included the effect of wall reflection of the transmitted energy back to the resonance region. These authors also showed that one hundred per cent mode conversion could occur for low field side incidence. 


\section{The Damping of the Fast Wave}

In the previous section the cold plasma model was used to obtain the Budden equation, which gives a good approximation to the propagation of the fast wave in the hybrid resonance region in a two-ion species plasma. We also discussed the generalisation to the triplet configuration when the fast wave cut-off on the high field side moves near to the hybrid resonance. For comparable ion densities the hybrid resonance is well separated from both cyclotron resonances and hence ion cyclotron damping is unimportant in the vicinity of the hybrid resonance. However, a wave launched from the low magnetic field side will cross one of the fundamental ion cyclotron resonances before reaching the hybrid resonance and may cross the other fundamental resonance before meeting the cut-off on the high field side. The wave may also encounter a second harmonic resonance of the heavier ion species. It is therefore important to calculate how much energy the fast wave will lose to the ions. If the energy absorbed by the electrons from the mode converted ion hybrid wave is to be maximised, then the ion damping of the fast wave should be minimized. It is also useful to include the electron dissipation terms for the fast wave. Although direct electron dissipation of the fast wave is weak for present experimental conditions, the direct electron dissipation becomes stronger in denser, hotter (ie high electron temperature) plasmas. In order to include all the electron dissipation terms in our second order description, we must extend the fast wave approximation to the full dielectric tensor and hence include the parallel electric field of the fast wave. This is accomplished as follows ${ }^{5}$. Writing out Eq (1) in full, we obtain

$$
\begin{gathered}
\left(\varepsilon_{x x}-n_{\|}^{2}\right) E_{x}+\varepsilon_{x y} E_{y}+\left(\varepsilon_{x z}+n_{\|} n_{\perp}\right) E_{z}=0 \\
-\varepsilon_{x y} E_{x}+\left(\varepsilon_{y y}-n_{\|}^{2}-n_{\perp}^{2}\right) E_{y}+\varepsilon_{y z} E_{z}=0 \\
\left(\varepsilon_{x z}+n_{\|} n_{\perp}\right) E_{x}-\varepsilon_{y z} E_{y}+\left(\varepsilon_{z z}-n_{\perp}^{2}\right) E_{z}=0
\end{gathered}
$$

Using $\mathrm{Eq}_{\mathrm{q}}(30)$ to express $E_{z}$ in terms of $E_{x}$ and $E_{y}$, we obtain

$$
E_{z}=-\frac{\left(n_{\|} n_{\perp}+\varepsilon_{x z}\right) E_{x}}{\left(\varepsilon_{z z}-n_{\perp}^{2}\right)}+\frac{\varepsilon_{y z} E_{y}}{\left(\varepsilon_{z z}-n_{\perp}^{2}\right)}
$$

The three coupled equations, $(28)-(30)$, treated locally, yield the dispersion relation given in $\mathrm{Eq}$ (2), which describes all possible modes of a hot, magnetized plasma. Some of these modes will have electric field polarizations which contain a significant parallel electric field. Here, we are concerned with the fast wave in the ion cyclotron range of frequencies, for which the refractive index $\left(n_{\perp}\right)_{F W}$ satisfies the condition $\left(n_{\perp}^{2}\right)_{F W} \ll \varepsilon_{z z}$. Hence, in $\mathrm{Eq}(31)$ we may neglect $n_{\perp}^{2}$ in the denominator. In the numerator we use the fast wave approximation and replace $n_{\perp}$ by $n_{\perp c}$ (the cold, nonresonant fast wave refractive index). With these approximations we can substitute Eq (31) into Eqs (28) and (29) to obtain a modified two by two system of equations for $E_{z}$ and $E_{y}$, in which the effect of the parallel electric field is included. After 
eliminating $E_{x}$ and taking the inverse Fourier transform, we obtain the desired fast wave equation including the effect of the parallel electric field. The equation is

$$
\frac{d^{2} E_{y}}{d x^{2}}+\frac{\omega^{2}}{c^{2}}\left[\varepsilon_{y y}-n_{\|}^{2}+\frac{\varepsilon_{y z}^{2}}{\varepsilon_{z z}}+\frac{\left(\varepsilon_{x y}+\frac{\varepsilon_{y z}\left(n_{\|} n_{\perp c}+\varepsilon_{x z}\right)}{\varepsilon_{z z}}\right)^{2}}{\left(\varepsilon_{x x}-n_{\|}^{2}-\frac{\left(n_{\|} n_{\perp c}+\varepsilon_{x z}\right)^{2}}{\varepsilon_{z z}}\right)}\right] E_{y}=0
$$

The coefficient of $E_{y}$ in Eq. (32) is the fast wave potential, which governs the propagation, damping, mode conversion and reflection of the fast wave. The first term of this potential, $\varepsilon_{y y}-n_{\|}^{2}+\varepsilon_{y z}^{2} / \varepsilon_{z z}$, describes the collisionless electron dissipation of the fast wave. The second term in the potential is the resonant part, which contributes the mode converted energy.

In order to use $\mathrm{Eq}(32)$ to calculate the ion damping, we must replace $n_{\perp}$ with $n_{\perp c}$, the fast wave approximation, in Eqs (3) - (5) and then substitute the resulting expressions for $\varepsilon_{x x}, \varepsilon_{y y}$ and $\varepsilon_{x y}$ into $\mathrm{Eq}(32)$. This incorporates fundamental ion cyclotron damping and second harmonic cyclotron damping of both ion species. In order to incorporate the electron damping effects we use

$$
\begin{gathered}
\varepsilon_{x z}=\sum_{j} \frac{\omega_{p j}^{2}}{\omega \Omega_{j}} \frac{k_{\perp}}{k_{\|}} \frac{e^{-\lambda_{j}}}{\lambda_{j}} \sum_{n=-\infty}^{\infty} n I_{n}\left(\lambda_{j}\right)\left[1+\zeta_{n j} Z\left(\zeta_{n j}\right)\right] \\
\varepsilon_{y z}=i \sum_{j} \frac{\omega_{p j}^{2}}{\omega \Omega_{j}} \frac{k_{\perp} e^{-\lambda_{j}}}{k_{\|}} \sum_{n=-\infty}^{\infty}\left(I_{n}-I_{n}^{\prime}\right)\left[1+\zeta_{n j} Z\left(\zeta_{n j}\right)\right] \\
\varepsilon_{z z}=1+\sum_{j} 2 \frac{\omega_{p j}^{2}}{\omega} \frac{e^{-\lambda_{j}}}{k_{\| l}^{2} v_{T j}^{2}} \sum_{n=-\infty}^{\infty}\left(\omega-n \Omega_{j}\right) I_{n}\left[1+\zeta_{n j} Z\left(\zeta_{n j}\right)\right]
\end{gathered}
$$

where $j$ represents a summation over species. Here, it is the electron terms which are required, in particular, the $n=0$, Landau resonance, which only occurs in $\varepsilon_{y z}$ and $\varepsilon_{z z}$. We have already noted the $n=0$ electron term in $\varepsilon_{y y}$, given in Eq. (10). The $\varepsilon_{x z}, \varepsilon_{y z}$ and $\varepsilon_{z z}$ are expanded to lowest order in $\lambda_{j}$ and the fast wave approximation is again made by replacing $k_{\perp}$ by $k_{\perp c}$.

We can use Eq. (32) to calculate the local direct dissipation rate of the fast wave by the electrons. For a region where ion cyclotron damping is negligible, we obtain the local value of $k_{\perp}$ from Eq. (32) and calculate $\operatorname{Im}\left(k_{\perp}\right)_{F W}$, giving

$$
\operatorname{Im}\left(k_{\perp}\right)_{F W} \simeq \frac{1}{2 k_{\perp c}} \frac{\omega_{p e}^{2}}{c^{2}} \frac{k_{\perp}^{2} v_{T e}^{2}}{2 \Omega_{e}^{2}} \pi^{1 / 2} \zeta_{o e} e^{-\zeta_{o e}^{2}}
$$

The only significant contributions to Eq. (36) come from $\varepsilon_{y y}$, the transit time damping, and $\varepsilon_{y z}^{2} / \varepsilon_{z z}$ the combination of the cross terms and Landau damping. The result 
given in Eq. (36) is consistent with the electron power dissipated

$$
\begin{aligned}
P_{e} & =\frac{1}{2} \operatorname{Re}\left(J \cdot E^{*}\right) \\
& =\frac{1}{2} \operatorname{Re}\left(\sigma_{y y}^{e}\right)\left|E_{y}\right|^{2}+\operatorname{Im}\left(\sigma_{y z}^{e}\right) \operatorname{Im}\left(E_{z} E_{y}^{*}\right)+\frac{1}{2} \operatorname{Re}\left(\sigma_{z z}^{e}\right)\left|E_{z}\right|^{2}
\end{aligned}
$$

The fast wave equation, $\mathrm{Eq}(32)$, can be written

$$
\frac{d^{2} E_{y}}{d x^{2}}+Q(x) E_{y}=0
$$

where the potential $Q(x)$ is given by inspection of $\mathrm{Eq}(32)$. The conservation relation given by Eqs (32) and (38) is

$$
\frac{d}{d x}\left(\operatorname{Im}\left(E_{y}^{*} \frac{d E_{y}}{d x}\right)\right)=-\left|E_{y}\right|^{2} \operatorname{Im} Q(x)
$$

The left-hand-side of $\mathrm{Eq}(39)$ represents the Poynting flux and the right-hand-side is the local power dissipated. Integration of $\mathrm{Eq}(39)$ over the whole interaction region gives the total power absorbed

$$
P_{a b s}=-\int_{x_{1}}^{x_{2}} \operatorname{Im} Q(x)\left|E_{y}\right|^{2} d x
$$

where $x_{1}$ and $x_{2}$ are on the low and high field sides of the interaction region. The expression for the power absorbed includes all the ion and electron collisionless dissipation mechanisms and the power mode converted at the two-ion hybrid resonance. It is worth noting that the fast wave energy directly dissipated by the electrons is also given by the electron terms in $\operatorname{Im} Q(x)$, ie $P_{a b s}^{e}=-\int_{x_{1}}^{x_{2}} \operatorname{Im} Q^{e}(x)\left|E_{y}\right|^{2} d x$. The electron power absorption calculated in this way is in agreement with Eq (37). The second order fast wave equation does not usually allow the power mode converted to be separated from the power dissipated by collisionless wave-particle resonance. However, we shall show that this can be done, to a good approximation, for the case of comparable ion concentrations due to the separation of the cyclotron and hybrid resonances. This will be demonstrated in Section VII, where the results obtained from the integration of $\mathrm{Eq}(32)$ are presented.

\section{The Damping of the Ion Hybrid Wave}

In Section IV we considered the optimum conditions to maximise the energy mode converted from the fast wave to the ion hybrid wave. In Section $V$ the competing ion and electron damping mechanisms were included in the fast wave equation to 
estimate how much power might be lost before the remaining energy reaches the mode conversion region. In this section we consider the fate of the mode converted ion hybrid wave as it propagates away from the two-ion hybrid resonance region. First we shall obtain a local dispersion relation, which we shall solve for the local damping rate of the ion hybrid wave. We shall then present results obtained from a toroidal, ray tracing code ${ }^{11}$ which allows the mode converted ion hybrid wave to be followed as it propagates away from the coupling region and its damping to be calculated.

The general dispersion relation for the ion hybrid wave in a hot, two ion species plasma with arbitrary ion concentrations is rather complicated, since the wave exists in both long wavelength, $k_{\perp}^{2} \rho_{j}^{2} \ll 1$, and short wavelength, $k_{\perp}^{2} \rho_{j}^{2}>1$, regimes, where $\rho_{j}$ is the Larmor radius of the $j$-th ion species. In the Appendix, we give the dispersion relation for arbitrary concentrations of two general ion species in the long wavelength regime. Here, we shall consider the specific case of a deuterium-tritium plasma in which the ion concentrations are equal and the temperatures are also assumed to be equal. Since we wish to calculate the electron dissipation of the ion hybrid wave, we start from the full, three by three dielectric tensor, but expand the relevant ion terms to first order in $\lambda_{j}$. Again, making use of the dominance of the $\varepsilon_{z z}$ element in the ion cyclotron range of frequencies, we approximate the full, three by three electromagnetic dispersion relation by

$$
n_{\perp}^{2}\left(\varepsilon_{x x}-n_{\|}^{2}\right)-\left(\varepsilon_{y y}^{i}-n_{\|}^{2}\right)\left(\varepsilon_{x x}-n_{\|}^{2}\right)-\varepsilon_{x y}^{2} \simeq\left(\frac{\varepsilon_{y z}^{2}}{\varepsilon_{z z}}+\varepsilon_{y y}^{e}\right)\left(\varepsilon_{x x}-n_{\|}^{2}\right)+\frac{n_{\|}^{2} n_{\perp}^{4}}{\varepsilon_{z z}}
$$

In the above equation we have only included the dominant electron damping terms. Note that the $\varepsilon_{y y}$ element has been separated explicitly into its ion and electron components. The first term on the right-hand-side of $\mathrm{Eq}(41)$ describes the electron dissipation of the fast wave and the second term the electron damping of the ion hybrid wave ${ }^{12}$. The fast wave damping term includes the contributions from transit time damping, $\varepsilon_{y y}^{e}$, electron Landau damping and the cross term contributions from these two effects. Since the ion hybrid wave is predominantly electrostatic away from the region of coupling to the fast wave, its dissipation is dominated by electron Landau damping. After a significant amount of algebra, the dispersion relation, to first order in the Larmor radius, can be written (see Appendix)

$$
\begin{gathered}
\left(\omega^{2}-c_{A}^{2} k_{\perp}^{2}\right)\left(\omega^{2}-\Omega_{i i}^{2}-\frac{\Omega_{T}^{2}}{4} N_{\|}^{2}+\frac{27}{200} \Omega_{T}^{2} \frac{k_{\perp}^{2} v_{T D}^{2}}{\Omega_{D}^{2}}\right)-\frac{9}{50} \Omega_{T}^{4}\left(\frac{1}{2}-\frac{25}{12} N_{\|}^{2}-2 \frac{k_{\perp}^{2} v_{T D}^{2}}{\Omega_{D}^{2}}\right) \\
\simeq\left(\frac{\Omega_{D}^{2}}{\omega_{p D}^{2}}\right)^{2} \frac{\left(\omega^{2}-\Omega_{D}^{2}\right)\left(\omega^{2}-\Omega_{T}^{2}\right) \omega^{2}}{\left(1+\frac{\omega_{T I}^{2}}{\omega_{p D}^{2}}\right)\left(1+\frac{\rho_{T}}{\rho D}\right) \Omega_{D}^{2} n_{\perp}^{4}} \frac{n_{z z}}{\varepsilon_{z z}}
\end{gathered}
$$

where only the term which dominates the damping of the ion hybrid wave has been retained on the right-hand-side of $\mathrm{Eq}$ (42). The numerical coefficients in this equation arise because of the specific choice of a deuterium-tritium plasma with equal concentrations and temperatures. The subscripts $D$ and $T$, of course, refer to deuterium and tritium quantities. For equal deuterium and tritium concentrations, $\Omega_{i i}^{2}=\Omega_{D} \Omega_{T}$. The remaining quantities in $\mathrm{Eq}(42)$ have already been defined. 
We shall now use $\mathrm{Eq}(42)$ to obtain the damping rate of the ion hybrid wave outside the region of coupling to the fast wave. In particular, we shall be concerned with the short wavelength region, where $c_{A}^{2} k_{\perp}^{2} / \omega^{2}>1$. Let us first solve Eq (42) perturbatively for the imaginary part of the frequency by assuming

$$
\omega \simeq \Omega_{I H}+\delta \omega
$$

where $\Omega_{I H}$ is the solution of the equation

$$
\omega^{2}-\Omega_{i i}^{2}-\frac{\Omega_{T}^{2}}{4} N_{\|}^{2}+\frac{27}{200} \Omega_{T}^{2} \frac{k_{\perp}^{2} v_{T D}^{2}}{\Omega_{D}^{2}}-\frac{9}{50} \Omega_{T}^{2} \frac{\left(\frac{1}{2}-\frac{25}{12} N_{\|}^{2}-\frac{k_{\perp}^{2} v_{2 D}^{2}}{\Omega_{D}^{2}}\right)}{\left(\Omega_{i i}^{2}-c_{A}^{2} k_{\perp}^{2}\right)}=0
$$

Substituting $\mathrm{Eq}_{\mathrm{q}}(43)$ into $\mathrm{Eq}(42)$ and extracting the imaginary part, we obtain

$$
\operatorname{Im} \delta \omega \simeq \frac{6}{50}\left(\frac{\Omega_{D}^{2}}{\omega_{p D}^{2}}\right)^{2} \frac{\left(\Omega_{I H}^{2}-\Omega_{D}^{2}\right)\left(\Omega_{I H}^{2}-\Omega_{T}^{2}\right)}{\Omega_{I H} \Omega_{i i}^{2}} \frac{\Omega_{I H}^{2}}{\Omega_{D}^{2}} \frac{n_{\|}^{2} n_{\perp}^{4}}{\left(N_{\perp}^{2}-1\right)} \frac{k_{\|}^{2} v_{T e}^{2}}{2 \omega_{p e}^{2}} \frac{\pi^{1 / 2} \zeta_{e} e^{-\zeta_{e}^{2}}}{\left|1+\zeta_{e} Z\left(\zeta_{e}\right)\right|^{2}}
$$

To a good approximation, $\Omega_{I H}^{2}$ can be replaced by $\Omega_{i i}^{2}$ in Eq (45). Clearly, $\operatorname{Im} \delta \omega<\theta$ since $\left(\Omega_{I H}^{2}-\Omega_{D}^{2}\right)\left(\Omega_{I H}^{2}-\Omega_{T}^{2}\right)<0$.

Next, we obtain the spatial damping rate. Since this will be compared with the numerical solution for a D- ${ }^{3} \mathrm{He}$ plasma in which $n_{3_{\mathrm{He}}} / n_{D}=\frac{1}{2}$ and $T_{3_{\mathrm{He}}}=T_{D}$, we require the corresponding equation to $\mathrm{Eq}(42)$ for this case. This can be written in the form (see Appendix)

$$
\begin{aligned}
\left(k_{\perp}^{2}-\frac{\omega^{2}}{c_{A}^{2}}\right)\left[k_{\perp}^{2}-\right. & \left.\frac{224}{9} \frac{\Omega_{D}^{2}}{v_{T D}^{2}} \frac{\left(\Omega_{i i}^{2}+\Omega_{3_{H e}}^{2} N_{\|}^{2} / 16-\omega^{2}\right)}{\Omega_{3_{H e}}^{2}}\right] \\
+ & \frac{2 \Omega_{3_{H e}}^{2}}{c_{A}^{2}} \frac{\Omega_{D}^{2}}{v_{T D}^{2}}\left(\frac{1}{7}-\frac{7}{12} N_{\|}^{2}-0.367 \frac{k_{\perp}^{2} v_{T D}^{2}}{\Omega_{D}^{2}}\right) \\
& =-\frac{24}{7}\left(\frac{\Omega_{D}^{2}}{\omega_{p D}^{2}}\right)^{2} \frac{\left(\omega^{2}-\Omega_{D}^{2}\right)}{v_{T D}^{2}} \frac{\left(\omega^{2}-\Omega_{3_{H e}}^{2}\right)}{c_{A}^{2}} \frac{\omega^{2}}{\Omega_{D}^{2}} \frac{n_{\|}^{2} n_{\perp}^{4}}{\varepsilon_{z z}}
\end{aligned}
$$

where $c_{A}^{2}=B_{0}^{2} /\left(\mu_{0} \sum_{j} n_{j} m_{j}\right)$ and $\Omega_{i i}^{2}=\frac{3}{4} \Omega_{3_{H e}}^{2}$ for this case. We can again solve Eq (46) perturbatively by assuming

$$
k_{\perp}=k_{+}+\delta k_{\perp}
$$

where $k_{ \pm}^{2}$ are the solutions of $\mathrm{Eq}(46)$ with the right-hand-side set to zero. $k_{+}^{2}$ corresponds to the ion hybrid wave and $k_{-}^{2}$ to the fast wave. In the short wavelength regime, $k_{+}^{2} \gg k_{-}^{2}$. Substituting $\mathrm{Eq}(47)$ into $\mathrm{Eq}(46)$, we obtain

$$
\begin{gathered}
I m \delta k_{\perp} \simeq \frac{24}{7}\left(\frac{\Omega_{D}^{2}}{\omega_{p D}^{2}}\right)^{2} \frac{\left(\omega^{2}-\Omega_{D}^{2}\right)}{v_{T D}^{2}} \frac{\left(\omega^{2}-\Omega_{3_{H e}}^{2}\right)}{c_{A}^{2}} \frac{\omega^{2}}{\Omega_{D}^{2}} \frac{n_{\|}^{2} n_{+}^{4}}{2 k_{+}\left(k_{+}^{2}-k_{-}^{2}\right)} \\
\times \frac{k_{\|}^{2} v_{T e}^{2}}{2 \omega_{p e}^{2}} \frac{\pi^{1 / 2} \zeta_{e} e^{-\zeta_{e}^{2}}}{1+\left.\zeta_{e} Z\left(\zeta_{e}\right)\right|^{2}}
\end{gathered}
$$


where $n_{+}$is $c k_{\perp} / \omega$ evaluated for $k_{\perp}=k_{+}$.

Notice that $I m \delta k_{\perp}<0$, since the ion hybrid mode is a backward wave. It can also be seen that the damping of the ion hybrid wave becomes stronger as it propagates towards shorter perpendicular wavelengths. Clearly, the ion hybrid wave will only be damped significantly by the electrons for $\left|\zeta_{e}\right| \lesssim 2$.

The approximate spatial damping rate given by $\mathrm{Eq}(48)$ is compared with the exact value obtained from the numerical solution of the full electromagnetic dispersion relation. This comparison is shown in Fig 3 where $I m n_{\perp}$ is plotted as a function of $\omega / k_{\|} v_{T e}$. The analytic approximation is in good agreement with the exact numerical result until $k_{\perp} \rho_{i}$ for the deuterons and tritons is no longer small as shown in Fig 4.

A more detailed picture of the propagation and damping of the ion hybrid wave can be obtained from a toroidal ray tracing code. The results of such a calculation for parameters appropriate to a JET discharge are shown in Figs 5-8. The electron density is $n_{e}=4 \times 10^{19} \mathrm{~m}^{-3}$, the magnetic field, $B_{o}=3.45$ tesla and $T_{e}=8 \mathrm{keV}$. The two ion species, are deuterium and a twenty five per cent concentration of helium-3 (relative to the electron density). The ion temperatures are $T_{D}=T_{3_{H e}}=5 \mathrm{keV}$. The trajectory of the mode converted ion hybrid wave in the poloidal plane is shown in Fig 5 . The wave can be seen to travel only a short distance poloidally before being damped by the electrons. The change of the parallel wave number as the ray propagates is shown in Fig 6. In Fig 7 the change of the modulus of the electric field of the ion hybrid wave as it propagates is given. The damping of the wave occurs fairly abruptly and results in complete absorption. The reason for the swift onset of damping is shown in Fig 8 , where the change in the resonant argument $\left|\zeta_{o e}\right|=\left|\omega / k_{\|} v_{T_{e}}\right|$ is shown. Due to the downshift, this quantity at first increases before reducing. Strong damping occurs as $\left|\zeta_{o e}\right|$ falls below 2 .

\section{Mode Conversion and Direct Damping}

In this section we present the results of integrating $\mathrm{Eq}(32)$ across the plasma. This enables us to assess the competition between direct dissipation of the fast wave and mode conversion at the two ion hybrid resonance for varying plasma conditions and different tokamaks. We shall give results for three different scenarios, the first appropriate to JET plasmas, the second is relevant to present TFTR plasmas and the third to denser, hotter, plasmas, also in TFTR.

The first set of results are given in Figs 9-12 and refer to the JET parameters used for the ray tracing results discussed in the previous section. The transmission $(T)$, reflection $(R)$ and total absorption $(D)$ coefficients plotted as a function of the parallel wave number are shown in Fig 9. The shape of the curves for $k_{\|}<5.5 \mathrm{~m}^{-1}$ is 
indicative of a Budden (resonance-cut-off) configuration, whereas the oscillations in $R$ and $D$ indicate that the high-field, fast wave cut-off is now present in the plasma, thus producing the triplet (cut-off-resonance-cut-off) configuration. Notice that the reflection coefficient drops to ten per cent for $k_{\|}=8.5 \mathrm{~m}^{-1}$. However, although this low reflection is associated with ninety per cent absorption, the curve does not indicate how this absorbed energy is divided between mode conversion and direct dissipation by the ions and electrons.

This question can be answered by referring to $\mathrm{Eq}(40)$ for the total absorption. The energy mode converted can be obtained from the fast wave equation by integrating $\operatorname{Im} Q(x)\left|E_{y}\right|^{2}$ only over the hybrid resonance. This provides a reliable guide to the mode converted fraction in this special case of comparable ion concentrations, where the ion cyclotron resonance regions are well separated from the two-ion hybrid resonance. This procedure is illustrated in Fig 10 in which the total absorption curve (A) is compared with the conversion integral (C), again plotted as a function of the parallel wave number. It can be seen that the fraction mode converted can be as high as eighty per cent under the optimum conditions, with ten per cent of the power dissipated outside the resonance region. The real and imaginary parts of the fast wave potential are shown in Figs 11 and 12. The real part shows the characteristic shape with the resonance on the high field side and the cut-off on the low field side. The imaginary part of the potential shows the two cyclotron resonances broadened by the Doppler spread of thermal ions and the central, very sharp, hybrid resonance. The imaginary part, which occurs as a weak background damping over the whole plasma, is of course, the direct electron fast wave damping.

A similar set of results is shown in Figs $13-16$ for TFTR parameters. Here, the electron density was taken as $5 \times 10^{19} \mathrm{~m}^{-3}$ and a deuterium-tritium plasma with fifty one per cent deuterium and forty nine per cent tritium was assumed. The magnetic field was taken as 4.8 tesla, the major radius $2.62 \mathrm{~m}$ and the electron and ion temperatures $10 \mathrm{keV}$ and $20 \mathrm{keV}$ respectively. The transmission, reflection and absorption coefficients shown in Fig 13 indicate the triplet behaviour for almost the whole range of the parallel wave number. The mode converted power can be as high as seventy five per cent, as shown in Fig 14. In this higher temperature case the damping outside the two-ion hybrid resonance region is stronger, but still only fifteen per cent. The curves for the real and imaginary parts of the potential, shown in Figs 15 and 16, are similar to the previous example, although the ion cyclotron resonances are broader due to the higher ion temperatures.

In the final example, given in Figs 17-20, the same TFTR parameters have been used, except the electron density is increased to $2 \times 10^{20} \mathrm{~m}^{-3}$ and the electron temperature taken as $30 \mathrm{keV}$, which represents an increase in the electron beta of a factor 12 . In this case, Fig 17 shows a behaviour of the transmission, reflection and absorption coefficients which is characteristic of the Budden configuration. The transmission is zero for the whole range of the parallel wave number and the total absorption always above seventy five per cent. Now, however, the mode converted energy, shown in 
Fig 18, is extremely small so that the absorption occurs on the low field side of the resonance. The real part of the potential, Fig 19, is similar to the other cases but the imaginary part of the potential, Fig 20, shows a greatly enhanced electron damping, with the hybrid resonance barely visible.

\section{Summary and Conclusions}

We have described the properties of ion Bernstein waves and the ion hybrid wave in a two ion species plasma. Whereas ion Bernstein waves are damped by ion cyclotron damping when $k_{\|}$is finite and $k_{\perp}$ is in the vicinity of the coupling region to the fast Alfvén wave (for low harmonics), the ion hybrid wave is not damped under these conditions. We have treated the mode conversion of the fast Alfven wave to an ion hybrid wave near the two-ion hybrid resonance. Both the Budden and triplet configuration have been discussed. In the latter case, one hundred per cent of the fast wave energy incident on the two-ion hybrid resonance-cut-off layer from the low field side can be mode converted. In general, the triplet configuration is the one most relevant to tokamak experiments. The effect of the parallel electric field has been included in the treatment of the problem. The fast wave equation contains all the collisionless loss mechanisms, including electron damping and mode conversion at the hybrid resonance. We have shown that the only damping mechanisms for the converted ion hybrid wave are due to the electrons, and an analytic approximation for the local, spatial damping rate has been derived. In addition, a ray tracing code shows that the ion hybrid wave can be completely damped by the electrons close to the ion hybrid resonance.

For future scenarios on large tokamaks, such as JET, TFTR and ITER, we have noted optimum conditions for mode conversion and have also quantified the requirement on the electron beta such that the fast Alfvén wave is absorbed directly by the electrons and no power is mode converted. Hence, the fast wave can be used to produce strong single pass electron heating regardless of the electron beta. For low electron beta, strong electron heating can occur via mode conversion, a result which has been convincingly demonstrated on TFTR ${ }^{3}$. For high beta, strong single pass electron heating by direct damping of the fast Alfvén wave can occur.

The understanding obtained of strong electron heating through mode conversion to the ion hybrid wave should enable a much more systematic investigation of fast wavelower hybrid synergy ${ }^{13}$ to be carried out. The study of non-inductive current drive by the mode converted ion hybrid waves has already begun on TFTR. The accessibility properties of the fast wave and the control of the absorption position through the two-ion hybrid resonance, make this a promising avenue for future investigations. Further theoretical analysis is clearly required. 


\section{Acknowledgements}

One of the authors (C N Lashmore-Davies) would like to thank Dr V P Bhatnagar for useful discussions. We would also like to thank Mr E Oliver who computed figures 1 and 2. This work was partly supported by the UK Department of Trade and Industry and Euratom, by the Centre canadien de fusion magnétique and by the US Department of Energy Contract No DE-FG02-91-ER-54109. CCFM is jointly managed and funded by Atomic Energy of Canada Limited, Hydro-Québec, and the Institut National de Recherche Scientifique. 


\section{Appendix A}

In this appendix we derive the finite Larmor radius dispersion relation for a plasma containing two ion species in comparable proportions. We start from Eq. (41), but neglect the electron damping terms on the right hand side, and substitute Eqs. (3)(5) for the dielectric tensor elements, in which the plasma dispersion functions for the ions are expanded asymptotically, assuming $\left(\omega-n \Omega_{j}\right) / k_{\|} v_{T j} \gg 1$ for both ion species. The dispersion relation is therefore relevant to the region of the two-ion hybrid resonance when this resonance is well separated from the cyclotron resonances of the two ion species. In other words, the dispersion relation is most accurate for the case when the two ion species are present in approximately equal proportions. Retaining terms to first order in $k_{\perp}^{2} \rho_{j}^{2}$ for both ion species, we multiply through Eq. (41) by

$$
\left(\frac{\Omega_{1}^{2}}{\omega_{p 1}^{2}}\right)^{2}\left(\Omega_{1}^{2}-\omega^{2}\right)\left(\omega^{2}-\Omega_{2}^{2}\right) \frac{\omega^{2}}{\Omega_{1}^{2}}\left(1+\frac{\omega_{p 2}^{2}}{\omega_{p 1}^{2}}\right)^{-1}\left(1+\frac{\rho_{2}}{\rho_{1}}\right)^{-1}
$$

and after some algebraic manipulation we obtain the dispersion relation

$$
\begin{aligned}
& \left(\omega^{2}-c_{A}^{2} k_{\perp}^{2}\right)\left\{\omega^{2}-\Omega_{i i}^{2}+\frac{N_{\|}^{2}\left(\frac{\omega^{2}}{\Omega_{1}^{2}}-1\right)\left(\omega^{2}-\Omega_{2}^{2}\right)\left(1+\frac{\rho_{2}}{\rho_{1}}\right)}{\left(1+\frac{\omega_{p 2}^{2}}{\omega_{p 1}^{2}}\right)}-\frac{1}{2\left(1+\frac{\omega_{p 2}^{2}}{\omega_{p 1}^{2}}\right)}\left[\omega^{2}-\Omega_{2}^{2}\right.\right. \\
& \left.\left.+\frac{\omega_{p 2}^{2}}{\omega_{p 1}^{2}}\left(\omega^{2}-\Omega_{1}^{2}\right) \frac{v_{T 2}^{2}}{v_{T 1}^{2}} \frac{\Omega_{1}^{2}}{\Omega_{2}^{2}}-\frac{\left(\omega^{2}-\Omega_{1}^{2}\right)\left(\omega^{2}-\Omega_{2}^{2}\right)}{\left(\omega^{2}-4 \Omega_{1}^{2}\right)}-\frac{\omega_{p 2}^{2}}{\omega_{p 1}^{2}} \frac{\left(\omega^{2}-\Omega_{1}^{2}\right)\left(\omega^{2}-\Omega_{2}^{2}\right)}{\left(\omega^{2}-4 \Omega_{2}^{2}\right)} \frac{v_{T 2}^{2}}{v_{T 1}^{2}} \frac{\Omega_{1}^{2}}{\Omega_{2}^{2}}\right] \frac{k_{\perp}^{2} v_{T 1}^{2}}{\Omega_{1}^{2}}\right\} \\
& =\frac{\omega^{2}}{\left(1+\frac{\omega_{p 2}^{2}}{\omega_{p 1}^{2}}\right)}\left\{\frac{\omega_{p 2}^{2}}{\omega_{p 1}^{2}} \frac{\left(\Omega_{1}-\Omega_{2}\right)^{2}}{\left(1+\frac{\rho_{2}}{\rho_{1}}\right)} \frac{\omega^{2}}{\Omega_{2}^{2}}+2 N_{\|}^{2}\left(\omega^{2}-\Omega_{2}^{2}\right)+2 N_{\|}^{2} \frac{\omega_{p 2}^{2}}{\omega_{p 1}^{2}}\left(\omega^{2}-\Omega_{1}^{2}\right)\right. \\
& +\frac{N_{\|}^{4}}{\Omega_{1}^{2}}\left(\omega^{2}-\Omega_{1}^{2}\right)\left(\omega^{2}-\Omega_{2}^{2}\right)\left(1+\frac{\rho_{2}}{\rho_{1}}\right)+N_{\|}^{2}\left(\frac{\omega^{2}}{\Omega_{1}^{2}}-1\right)\left(\omega^{2}-\Omega_{2}^{2}\right)\left(1+\frac{\rho_{2}}{\rho_{1}}\right) \\
& -\left[\frac{\left(\omega^{2}-\Omega_{2}^{2}\right)}{\left(1+\frac{\rho_{2}}{\rho_{1}}\right)}+\frac{\omega_{p 2}^{2}}{\omega_{p 1}^{2}} \frac{\left(\omega^{2}-\Omega_{1}^{2}\right)}{\left(1+\frac{\rho_{2}}{\rho_{1}}\right)}+\frac{N_{\|}^{2}\left(\omega^{2}-\Omega_{1}^{2}\right)\left(\omega^{2}-\Omega_{2}^{2}\right)}{\Omega_{1}^{2}}\right]\left[\frac{2 \Omega_{1}^{2}}{\left(\omega^{2}-\Omega_{1}^{2}\right)}\right. \\
& +\frac{2 \omega_{p 2}^{2}}{\omega_{p 1}^{2}} \frac{\Omega_{1}^{2}}{\left(\omega^{2}-\Omega_{1}^{2}\right)} \frac{v_{T 2}^{2}}{V_{T 1}^{2}} \frac{\Omega_{1}^{2}}{\Omega_{2}^{2}}-\frac{\Omega_{1}^{2}}{\left(\omega^{2}-4 \Omega_{1}^{2}\right)}-\frac{\omega_{p 2}^{2}}{\omega_{p 1}^{2}} \frac{\Omega_{1}^{2}}{\left(\omega^{2}-4 \Omega_{2}^{2}\right)} \frac{v_{T 2}^{2}}{v_{T 1}^{2}} \frac{\Omega_{1}^{2}}{\Omega_{2}^{2}}-\frac{\Omega_{1}^{2}}{\omega^{2}} \\
& \left.-\frac{\omega_{p 2}^{2}}{\omega_{p 1}^{2}} \frac{\Omega_{1}^{2}}{\omega^{2}} \frac{v_{T 2}^{2}}{v_{T 1}^{2}} \frac{\Omega_{1}^{2}}{\Omega_{2}^{2}}\right] \frac{k_{\perp}^{2} v_{T 1}^{2}}{\Omega_{1}^{2}}-2\left[\frac{\left(\omega^{2}-\Omega_{2}^{2}\right) \omega}{\left(1+\frac{\rho_{2}}{\rho_{1}}\right) \Omega_{1}}+\frac{\omega_{p 2}^{2}}{\omega_{p 1}^{2}} \frac{\left(\omega^{2}-\Omega_{1}^{2}\right) \omega}{\left(1+\frac{\rho_{2}}{\rho_{1}}\right) \Omega_{2}}\right]\left[-\frac{\Omega_{1}^{2}}{\left(\omega^{2}-\Omega_{1}^{2}\right)} \frac{\Omega_{1}}{\omega}\right.
\end{aligned}
$$




$$
\begin{gathered}
\left.-\frac{\omega_{p 2}^{2}}{\omega_{p 1}^{2}} \frac{\Omega_{1}^{2}}{\left(\omega^{2}-\Omega_{2}^{2}\right)} \frac{\Omega_{2}}{\omega} \frac{v_{T 2}^{2}}{v_{T 1}^{2}} \frac{\Omega_{1}^{2}}{\Omega_{2}^{2}}+\frac{\Omega_{1}^{2}}{\left(\omega^{2}-4 \Omega_{1}^{2}\right)} \frac{\Omega_{1}}{\omega}+\frac{\omega_{p 2}^{2}}{\omega_{p 1}^{2}} \frac{\Omega_{1}^{2}}{\left(\omega^{2}-4 \Omega_{2}^{2}\right)} \frac{\Omega_{2}}{\omega} \frac{v_{T 2}^{2}}{v_{T 1}^{2}} \frac{\Omega_{1}^{2}}{\Omega_{2}^{2}}\right] \frac{k_{\perp}^{2} v_{T 1}^{2}}{\Omega_{1}^{2}} \\
+\frac{1}{2}\left[\omega^{2}-\Omega_{2}^{2}+\frac{\omega_{p 2}^{2}}{\omega_{p 1}^{2}}\left(\omega^{2}-\Omega_{1}^{2}\right) \frac{v_{T 2}^{2}}{v_{T 1}^{2}} \frac{\Omega_{1}^{2}}{\Omega_{2}^{2}}-\frac{\left(\omega^{2}-\Omega_{1}^{2}\right)\left(\omega^{2}-\Omega_{2}^{2}\right)}{\left(\omega^{2}-4 \Omega_{1}^{2}\right)}\right. \\
\left.\left.-\frac{\omega_{p 2}^{2}}{\omega_{p 1}^{2}} \frac{\left(\omega^{2}-\Omega_{1}^{2}\right)\left(\omega^{2}-\Omega_{2}^{2}\right)}{\left(\omega^{2}-4 \Omega_{2}^{2}\right)} \frac{v_{T 2}^{2}}{v_{T 1}^{2}} \frac{\Omega_{1}^{2}}{\Omega_{2}^{2}}\right] \frac{k_{1}^{2} v_{T 1}^{2}}{\Omega_{1}^{2}}\right\}
\end{gathered}
$$

We reiterate that $c_{A}^{2}=\frac{B_{0}^{2}}{\mu_{0}\left(\rho_{1}+\rho_{2}\right)}$ and $N_{\|}^{2}=c_{A}^{2} k_{\|}^{2} / \omega^{2}$. The dispersion relation given in Eq. (A.1) includes both the ion hybrid wave and the fast wave. The specific dispersion relations given in Eqs. (42) and (46) were obtained from Eq. (A.1) with the addition of the second term on the right-hand-side of Eq. (41) which describes the damping of the ion hybrid wave. 


\section{References}

${ }^{1} \mathrm{~A}$ K Ram, A Bers and V Fuchs, Proceedings of the 20th EPS Conference on Controlled Fusion and Plasma Physics, Vol III, Lisboa, Portugal, 1993, eds J A Costa Cabral, M E Manso, F M Serra and F C Schüller, pp 897-900.

${ }^{2}$ J Jacquinot, B D McVey and J E Scharer, Phys Rev Letters 29, 88 (1977).

${ }^{3} \mathrm{R}$ Majeski, C K Phillips and J R Wilson, Phys Rev Letters 73, 2204 (1994); R Majeski, J C Hosea, C K Phillips, J H Rogers, G Schilling, J E Stevens and J R Wilson, 10th Topical Conference on Radio Frequency Power in Plasmas 1993, Boston, AIP Conference Proceedings No 289, edited by M Porkolab and J Hosea (American Institute of Physics, New York, 1993) p 401.

${ }^{4} \mathrm{~K}$ G Budden, in The Propagation of Radio Waves (Cambridge University Press, Cambridge, 1985) p 596.

${ }^{5} \mathrm{~V}$ Fuchs, A K Ram, S D Schultz, A Bers and C N Lashmore-Davies, Phys Plasmas 2, 1637 (1995).

${ }^{6} \mathrm{~T}$ H Stix, in Waves in Plasmas (American Institute of Physics, New York, 1992) $\mathrm{p}$ 257.

${ }^{7}$ A I Akhiezer, I A Akhiezer, R V Polovin, A G Sitenko and K N Stepanov, in Plasma Electrodynamics (Pergamon Press, Oxford, 1975) Vol I p 284.

${ }^{8} \mathrm{C}$ K Chow, A K Ram and A Bers, Phys Fluids B1, 2018 (1989).

${ }^{9} \mathrm{~B}$ Saoutic, A Bécoulet, T Hutter, D Fraboulet, A K Ram and A Bers, Phys Rev Letters 76, 1647 (1996).

${ }^{10}$ Y C Ngan and D G Swanson, Phys Fluids 20, 1920 (1977).

${ }^{11}$ A K Ram and A Bers, Phys Fluids B3, 1059 (1991).

${ }^{12}$ M Ono, Phys Fluids B5, 241 (1993).

${ }^{13}$ The JET Team (presented by C Gormezano), in Plasma Physics and Controlled Nuclear Fusion Research, 1992, Würzburg, (International Atomic Energy Agency, Vienna, 1993) Vol I p 587. 


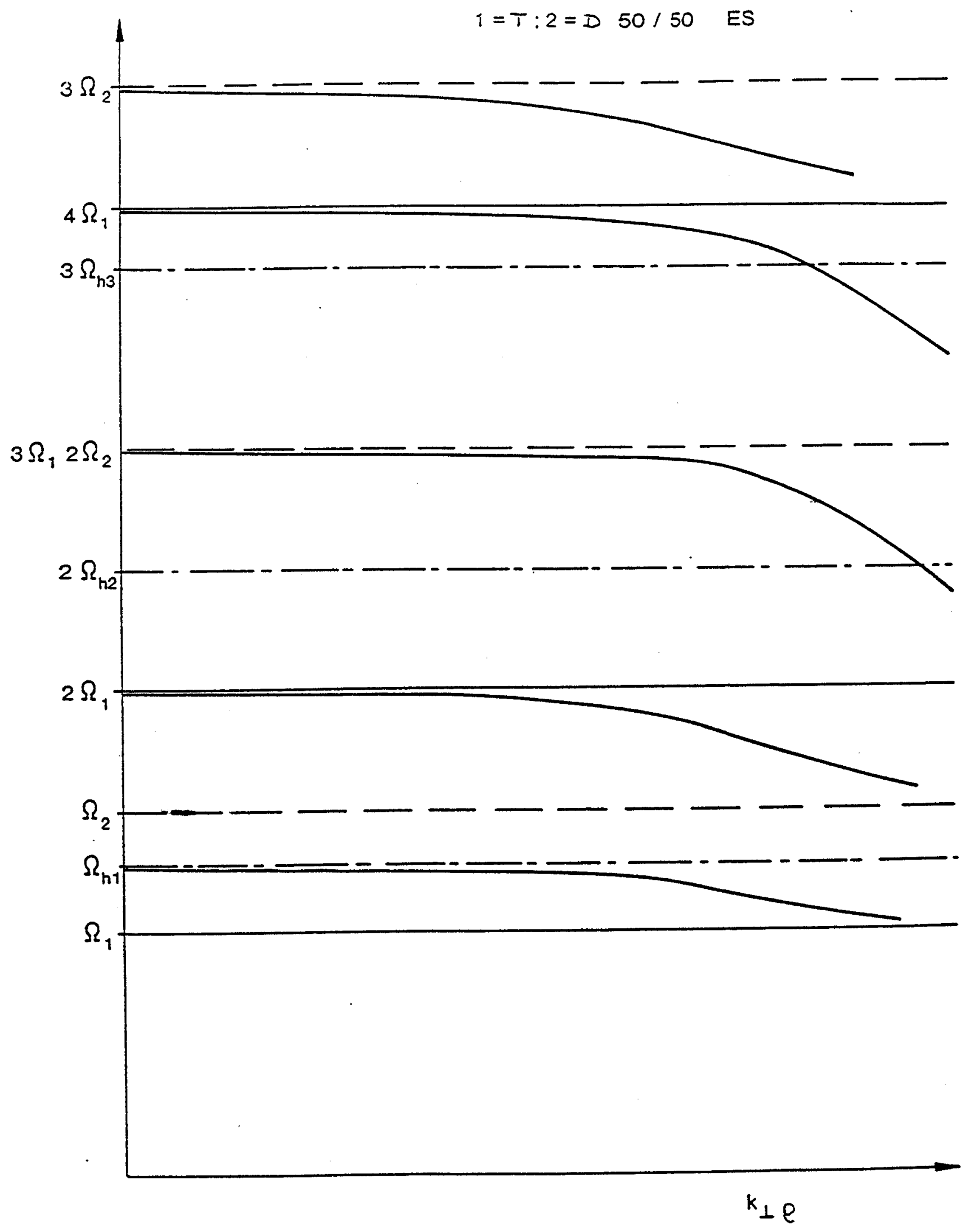

Figure 1 The electrostatic dispersion relation for ion Bernstein waves propagating at right angles to a uniform magnetic field in a plasma with equal concentrations of deuterium and tritium. The deuterium concentration, $n_{D}=2.5 \times 10^{19} \mathrm{~m}^{-3}$, the magnetic field, $B_{o}=3$ tesla and the ion temperatures are both taken as $10 \mathrm{keV}$. 

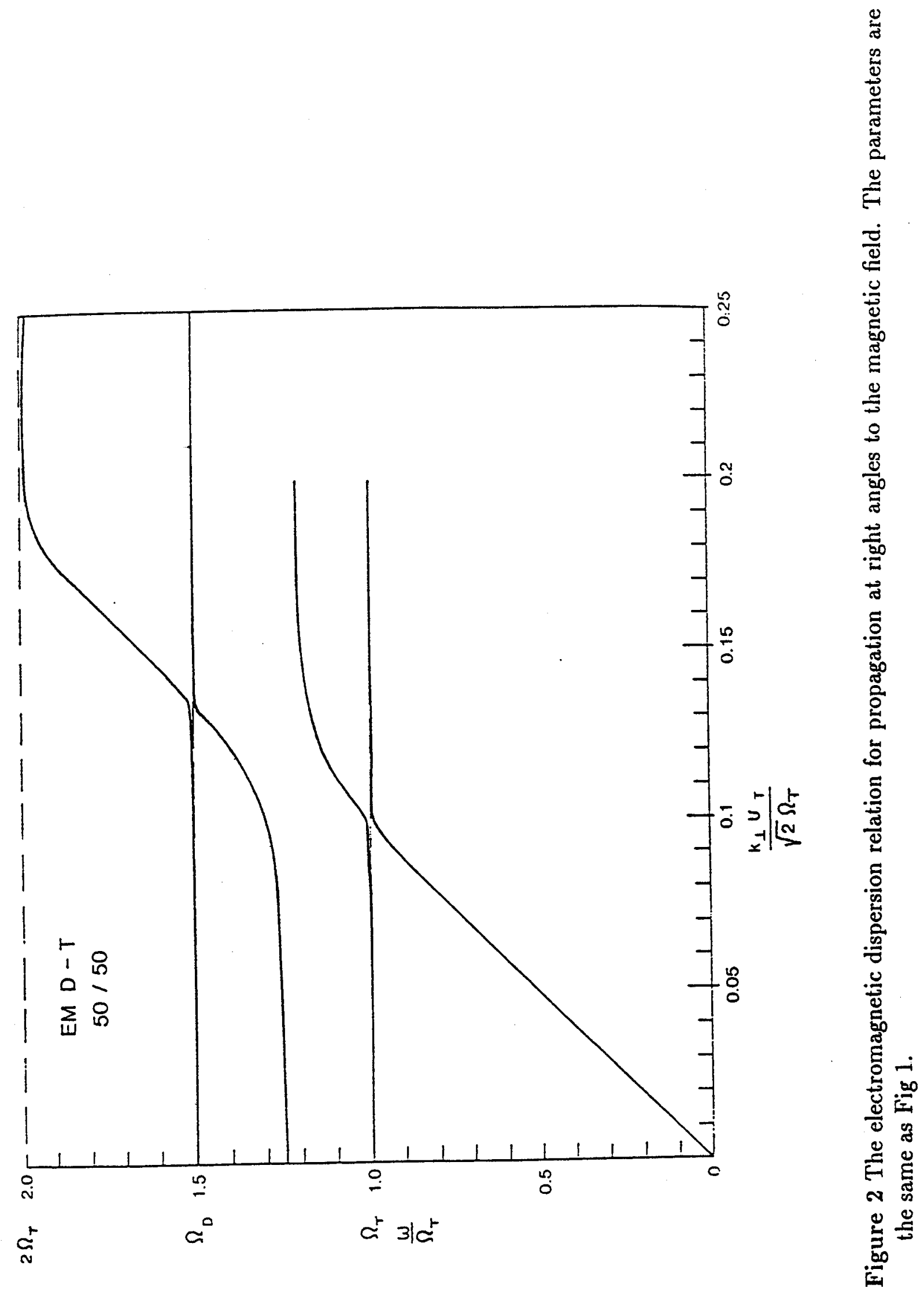


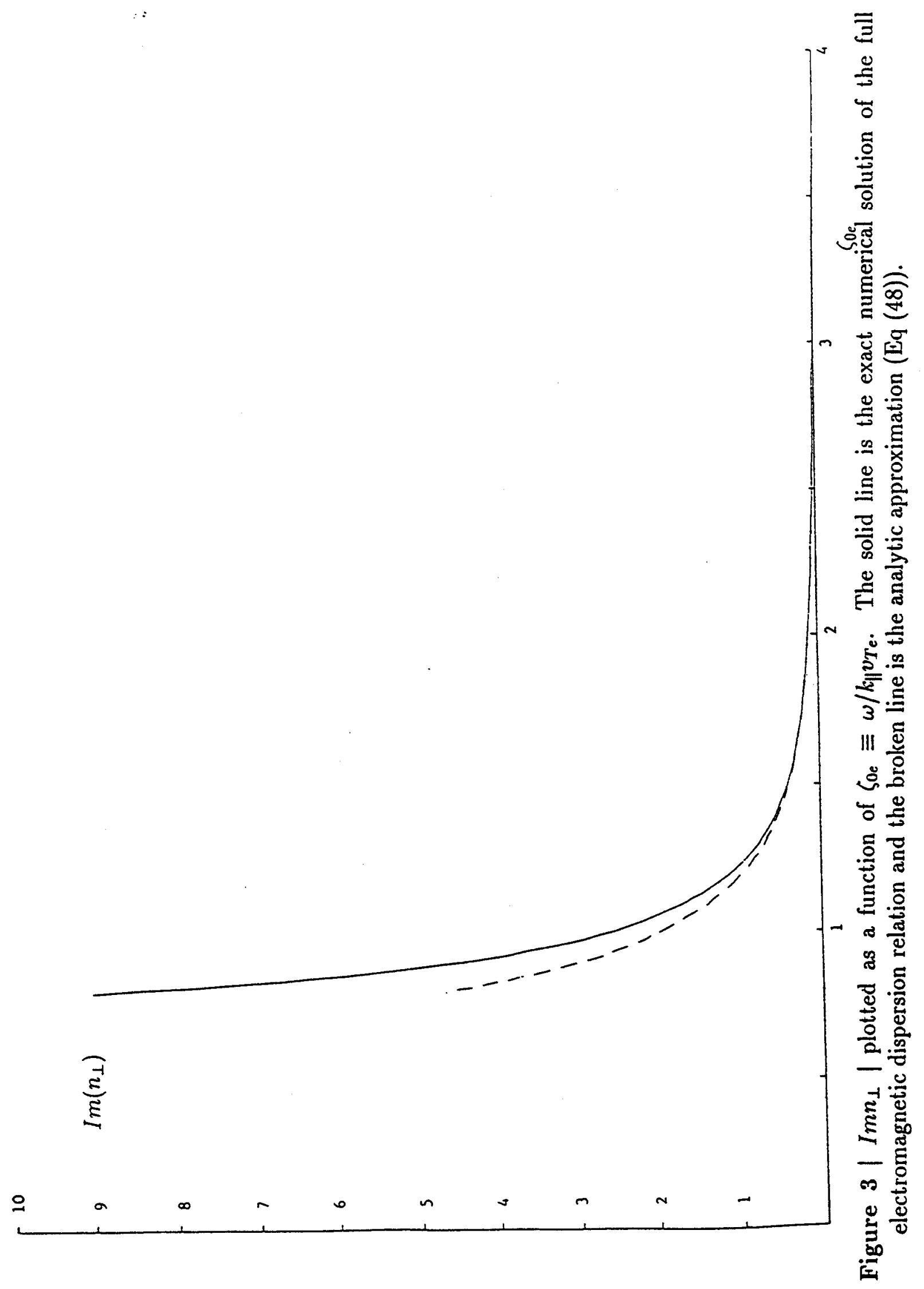




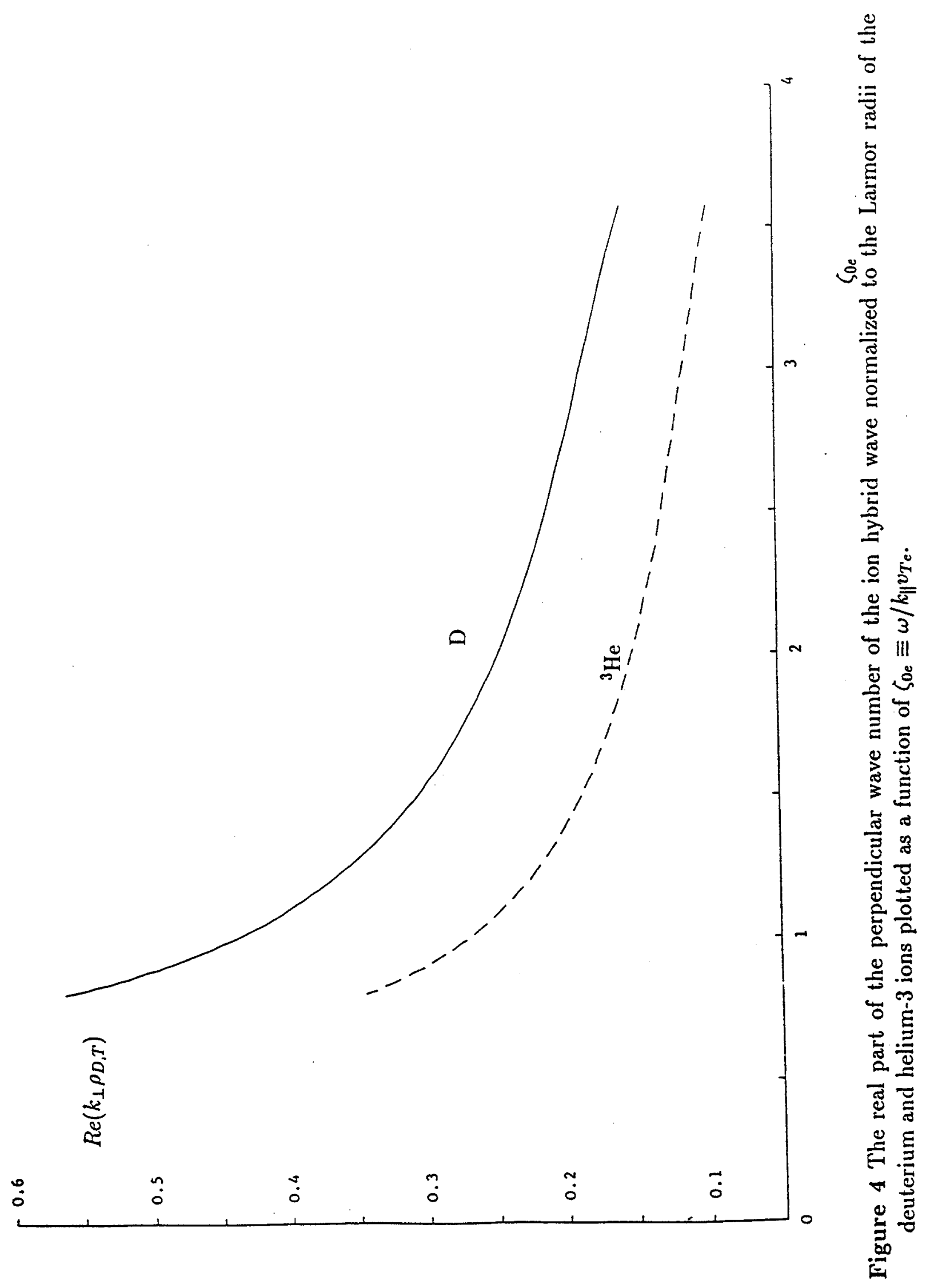




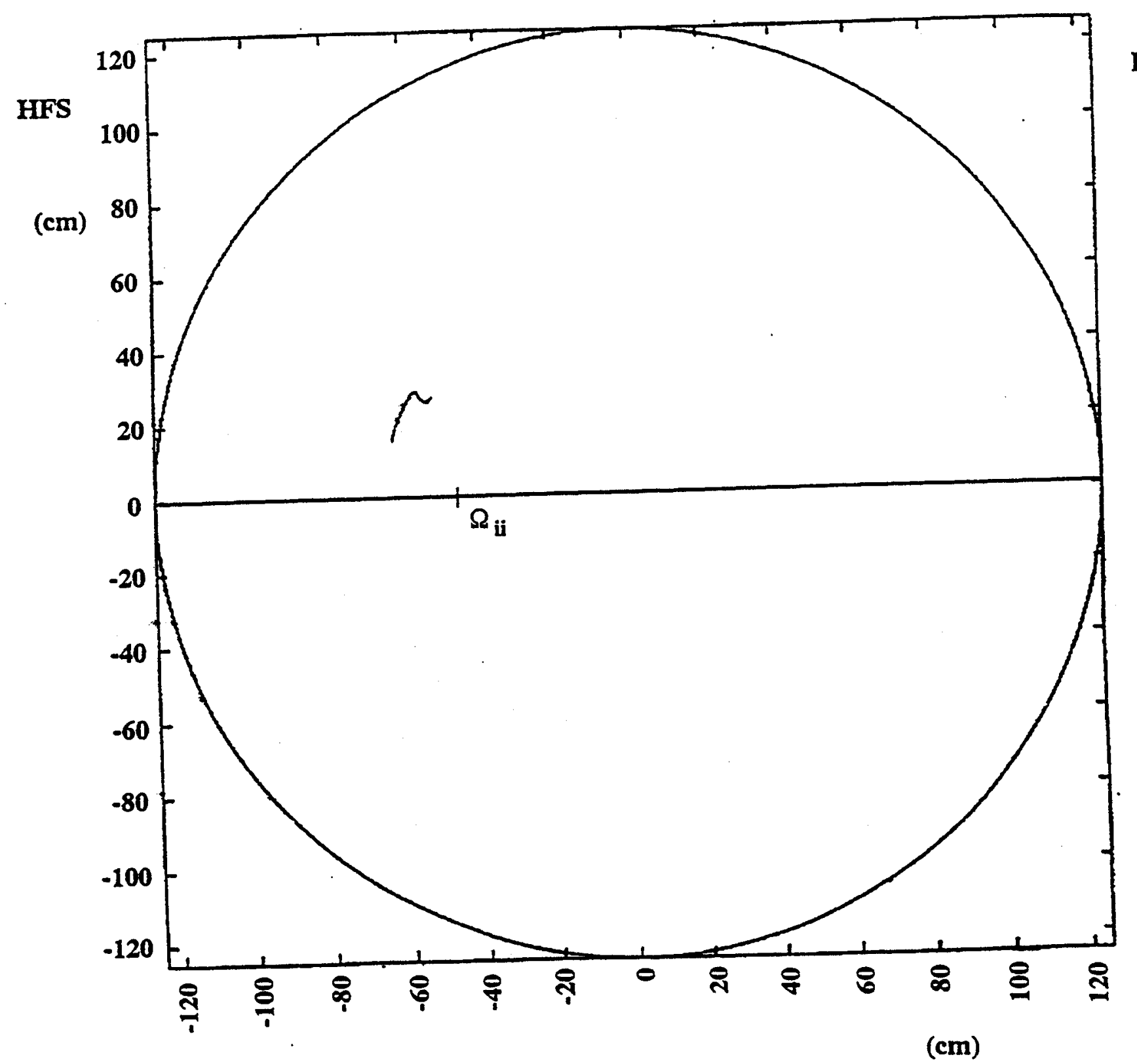

LFS

Figure 5 Poloidal projection of the mode converted ion hybrid wave. The parameters are appropriate to JET: $n_{e}=4 \times 10^{19} \mathrm{~m}^{-3}, B_{0}=3.45$ tesla, deuteriumhelium 3 plasma with $25 \%$ helium 3 ( relative to the electron density), $T_{e}=8$ $\mathrm{keV}, T_{D}=T_{3_{H e}}=5 \mathrm{keV}$. 


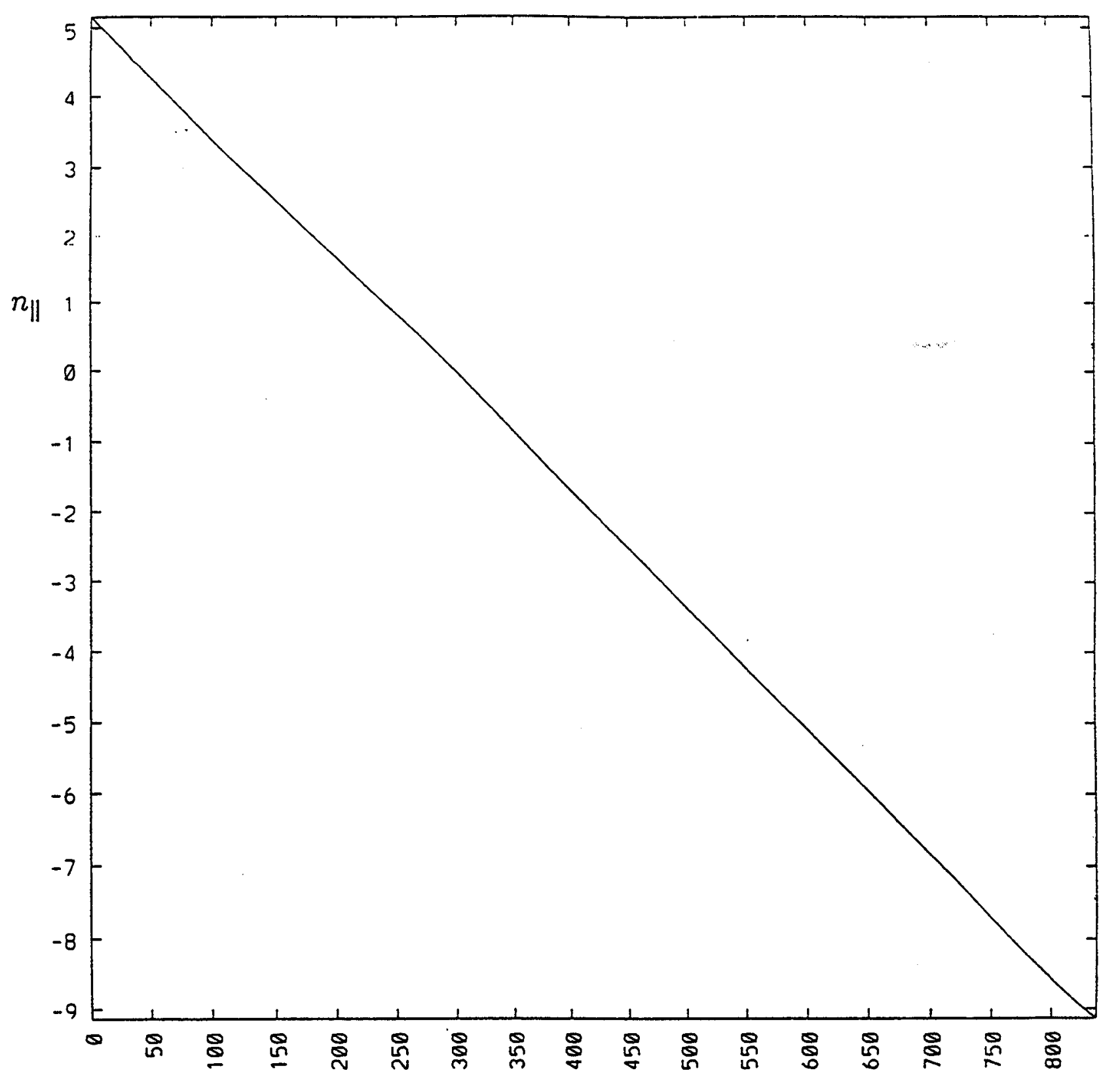

N-PARALLEL VERSUS TIME

Figure 6 Change of the parallel refractive index along the ion hybrid ray. The parameters are the same as Fig 3. 


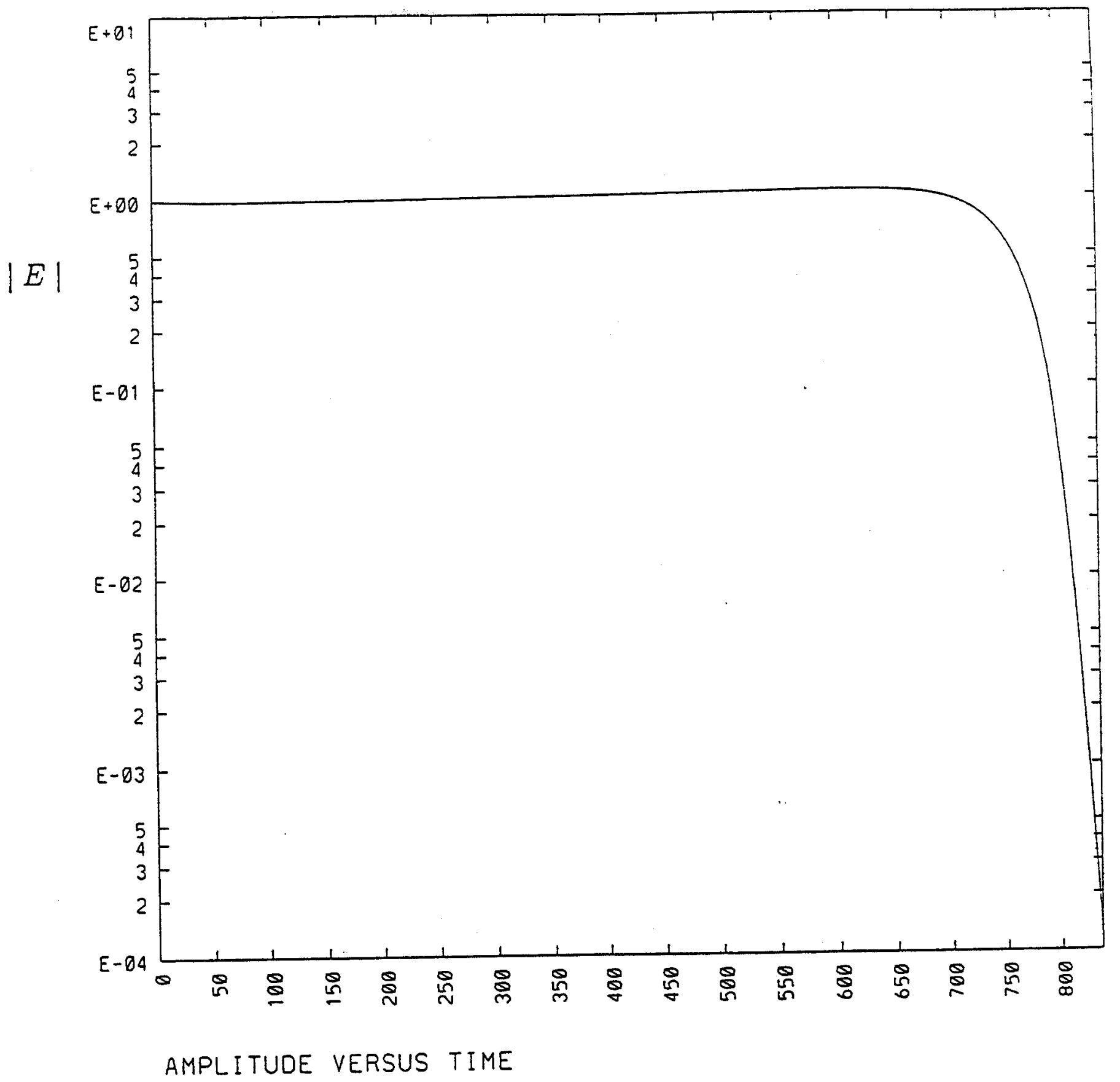

Figure 7 The change in the modulus of the electric field of the ion hybrid wave along the ray trajectory. The parameters are the same as Fig 3. 


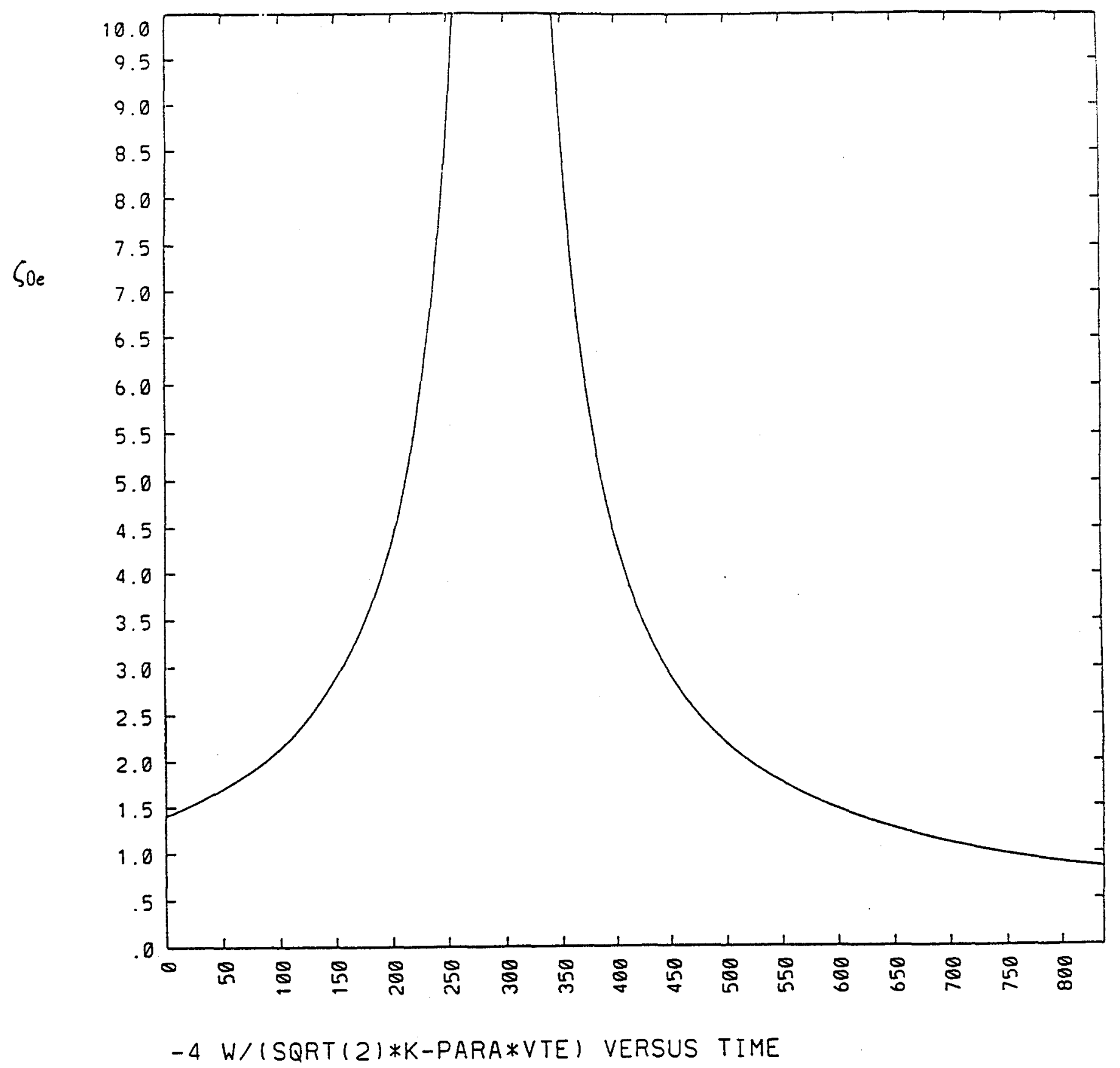

Figure 8 The change in the normalized parallel phase speed $\zeta_{o e}=\omega / k_{\|} v_{T e}$ along the ion hybrid ray trajectory. The parameters are the same as Fig 3. 


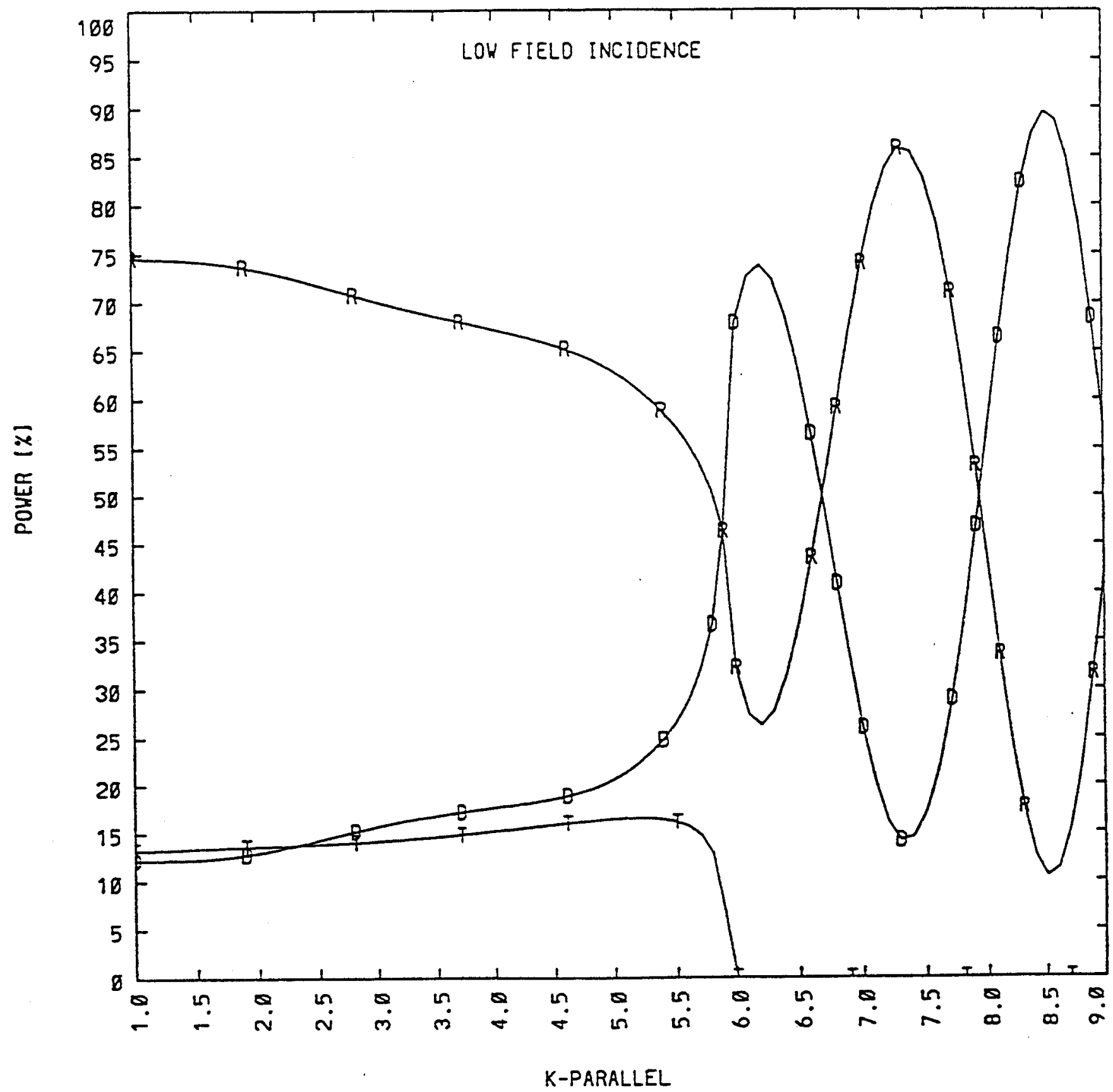

Figure 9 Transmission $(T)$, reflection $(R)$ and total absorption $(D)$ fractions plotted as a function of the parallel wave number for JET conditions: $n_{e}=4 \times$ $10^{19} \mathrm{~m}^{-3}, B_{0}=3.45$ tesla, $R=2.9 \mathrm{~m}$ for a $\mathrm{D}\left({ }^{3} \mathrm{He}\right)$ plasma with a helium- 3 concentration of $25 \%, T_{e}=8 \mathrm{keV}$ and $T_{D}=T_{3_{\mathrm{He}}}=5 \mathrm{keV}$. 


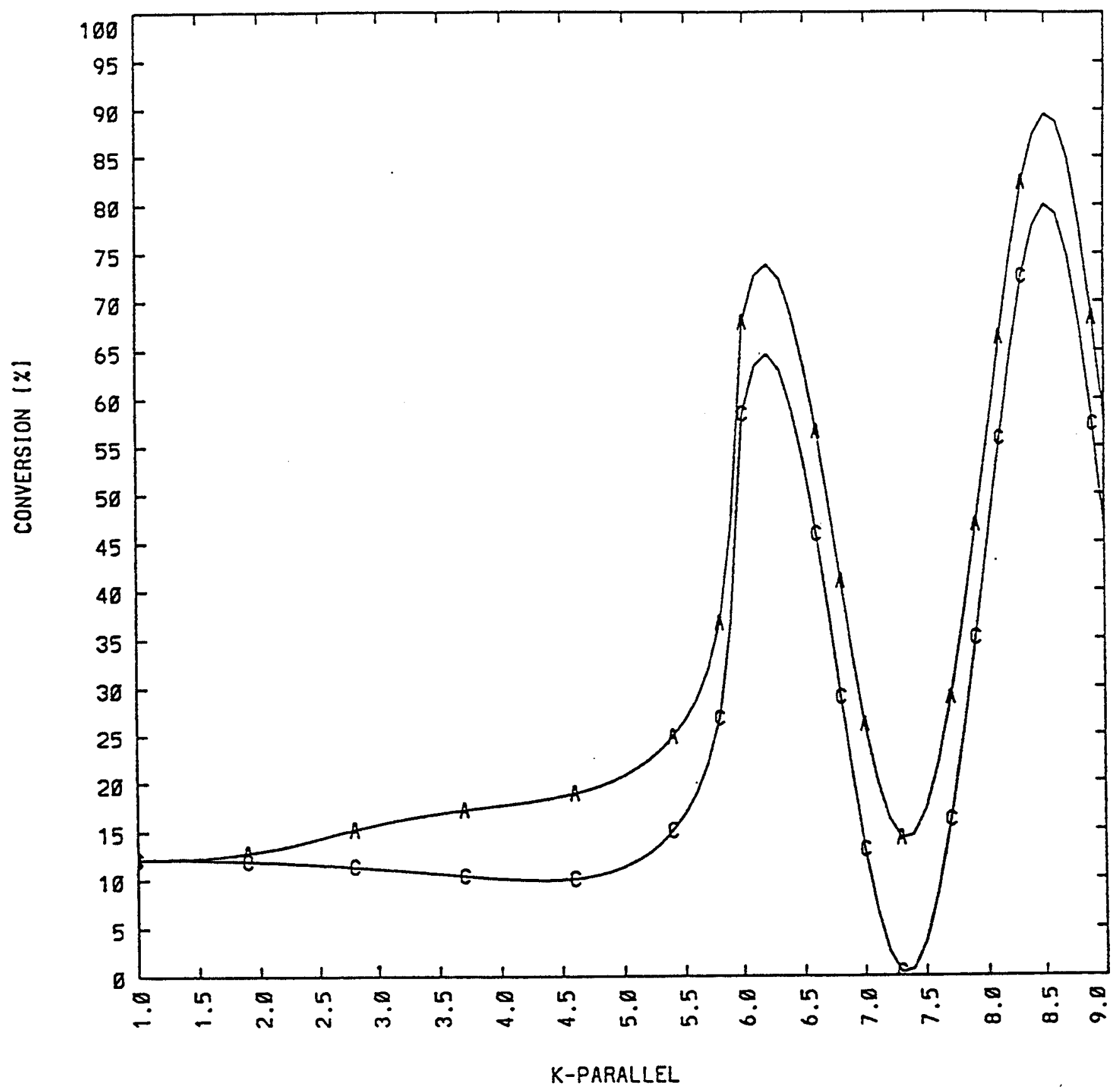

Figure 10 Comparison of total absorption (A) with the mode converted power (C) plotted as a function of the parallel wave number. The parameters are the same as in Fig 9. 


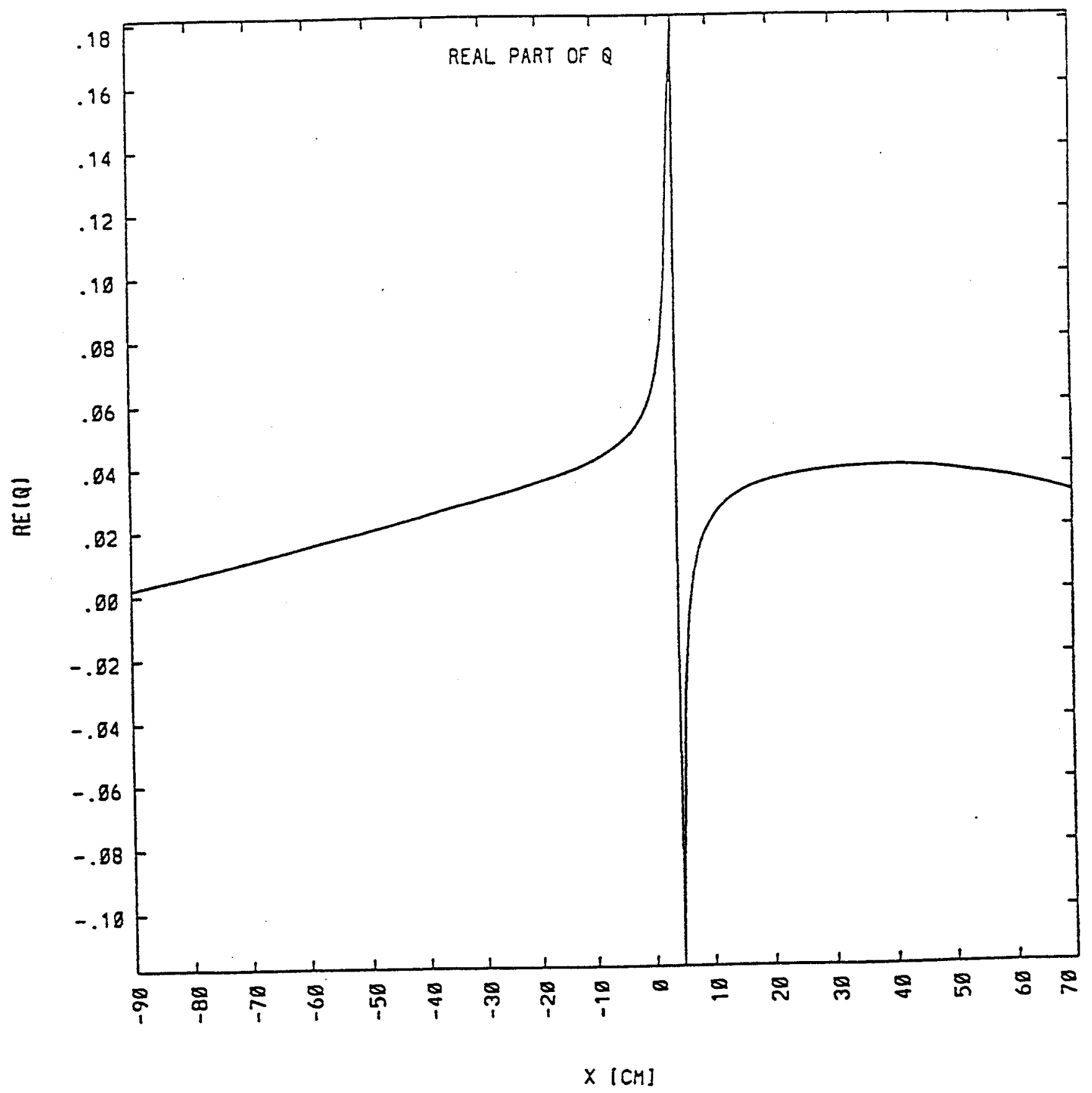

Figure 11 The real part of the fast wave potential (Eq (32)) plotted as a function of the spatial co-ordinate. The parameters are the same as Fig 9 with $k_{\|}=5 \mathrm{~m}^{-1}$. 


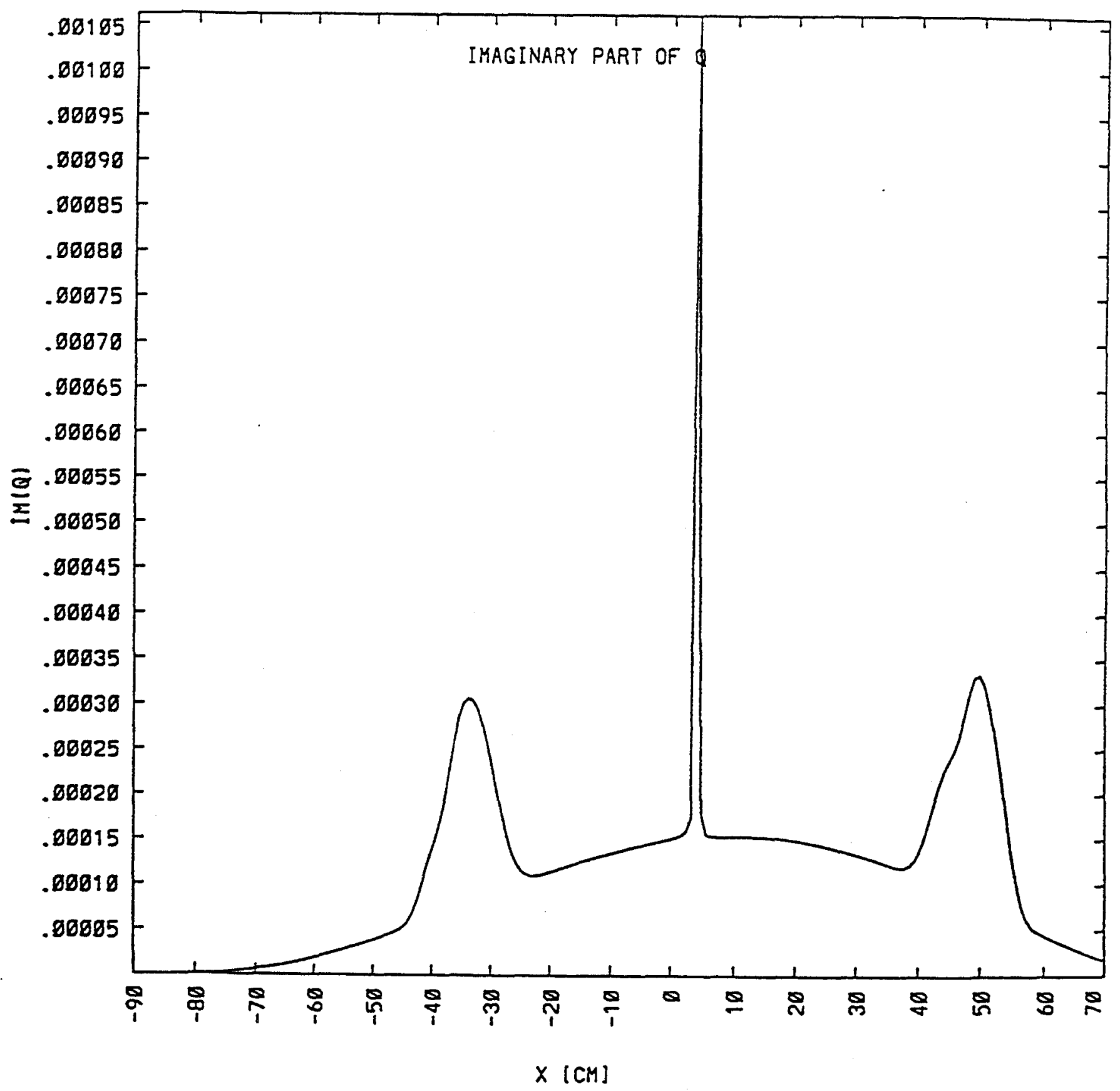

FIgure 12 The imaginary part of the fast wave potential plotted as a function of the spatial co-ordinate. The parameters are the same as Fig 9 with $k_{\|}=5 \mathrm{~m}^{-1}$. 


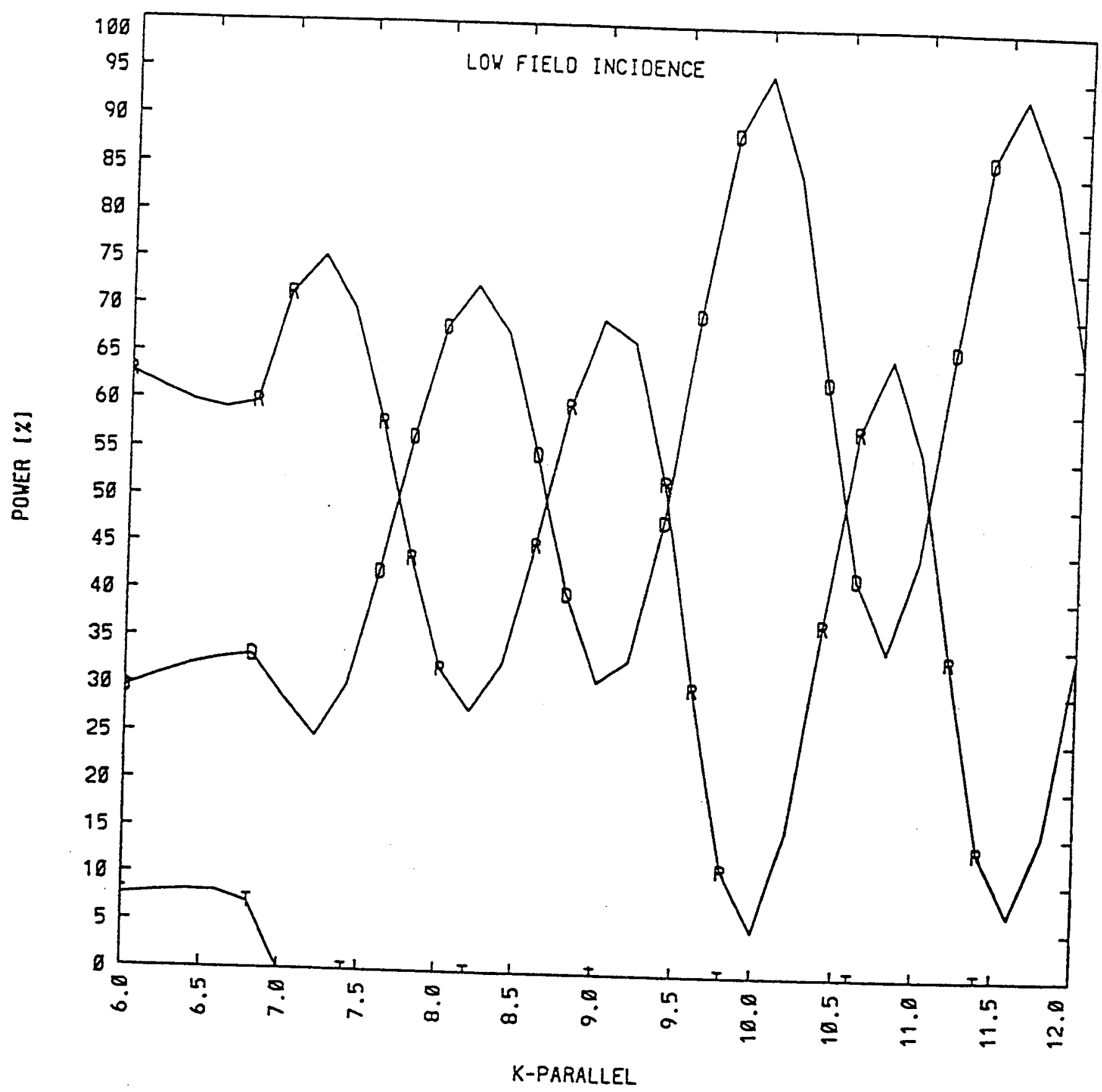

Figure 13 Transmission $(T)$, reflection $(R)$ and total absorption $(D)$ fractions plotted as a function of the parallel wavenumber for TFTR parameters: $n_{e}=$ $5 \times 10^{19} \mathrm{~m}^{-3}, B_{\circ}=4.8$ tesla, $R=2.62 \mathrm{~m}, T_{\mathrm{e}}=10 \mathrm{keV}$ for a deuterium (51\%) tritium (49\%) plasma with $T_{D}=T_{T}=20 \mathrm{keV}$. 


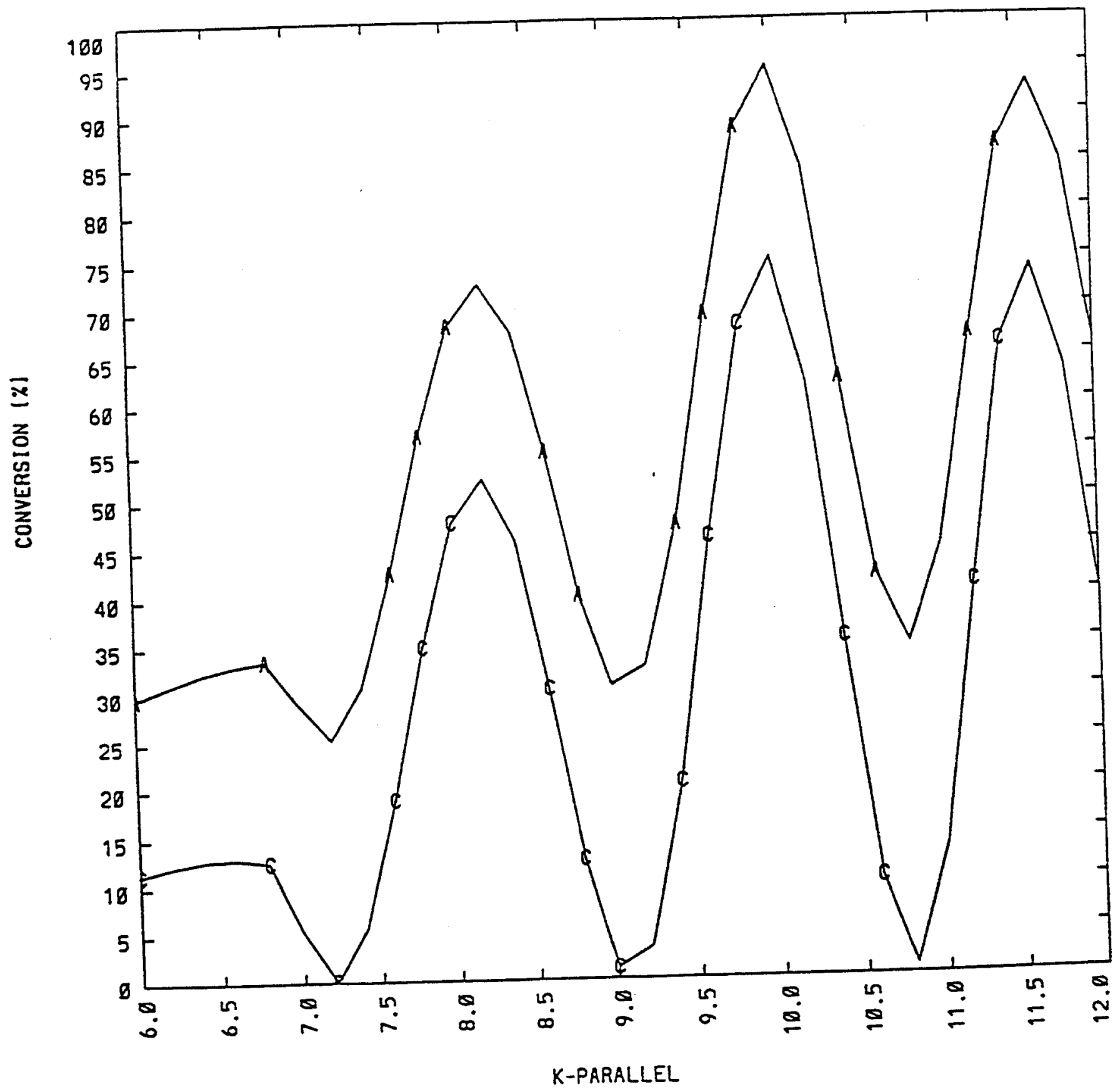

Figure 14 Comparison of total absorption (A) with the mode converted power (C) plotted as a function of the parallel wave number. The parameters are the same as Fig 13. 


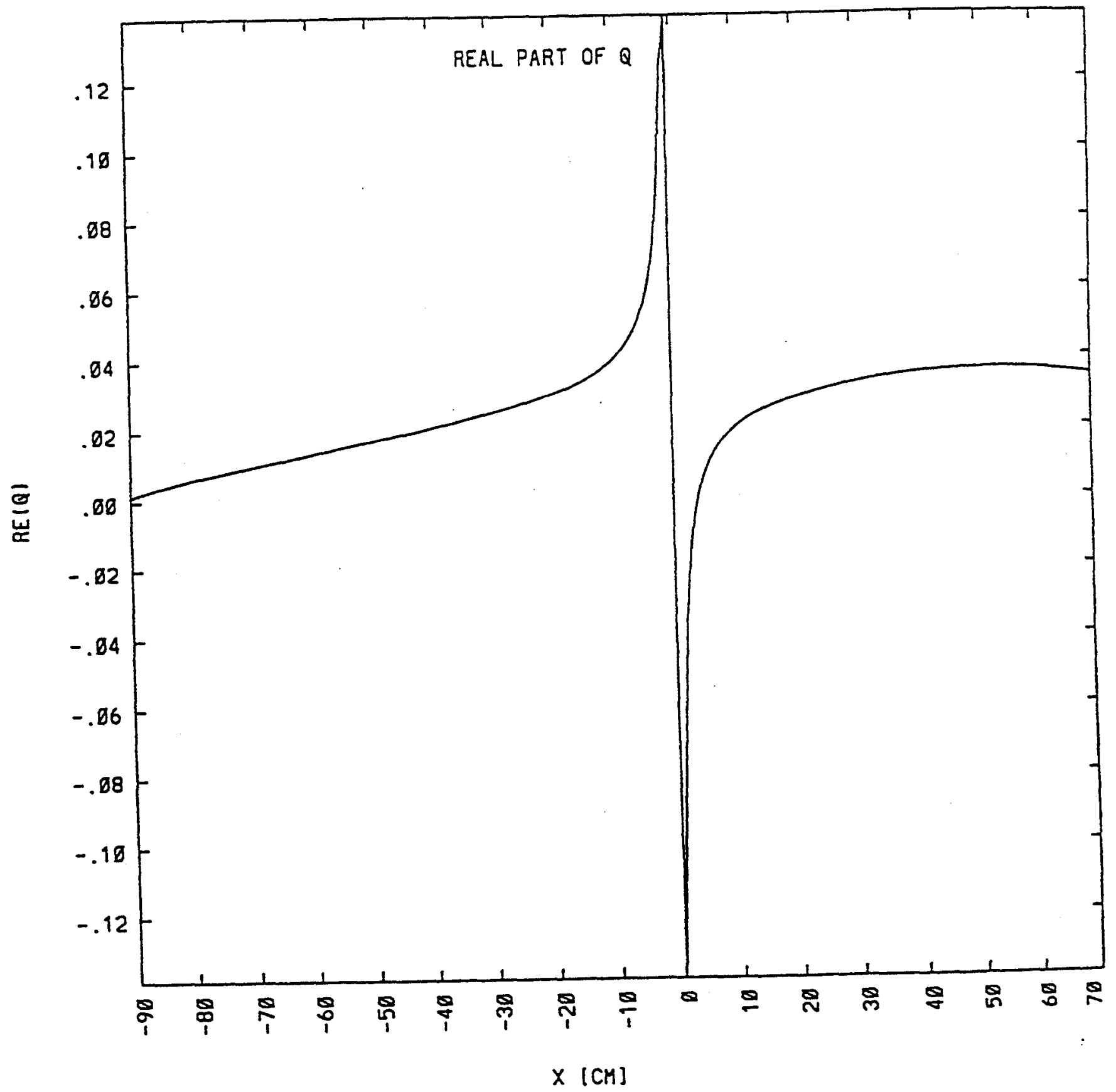

Figure 15 The real part of the fast wave potential plotted as a function of the spatial co-ordinate. The parameters are the same as Fig 13 with $k_{\|}=6.6 \mathrm{~m}^{-1}$. 


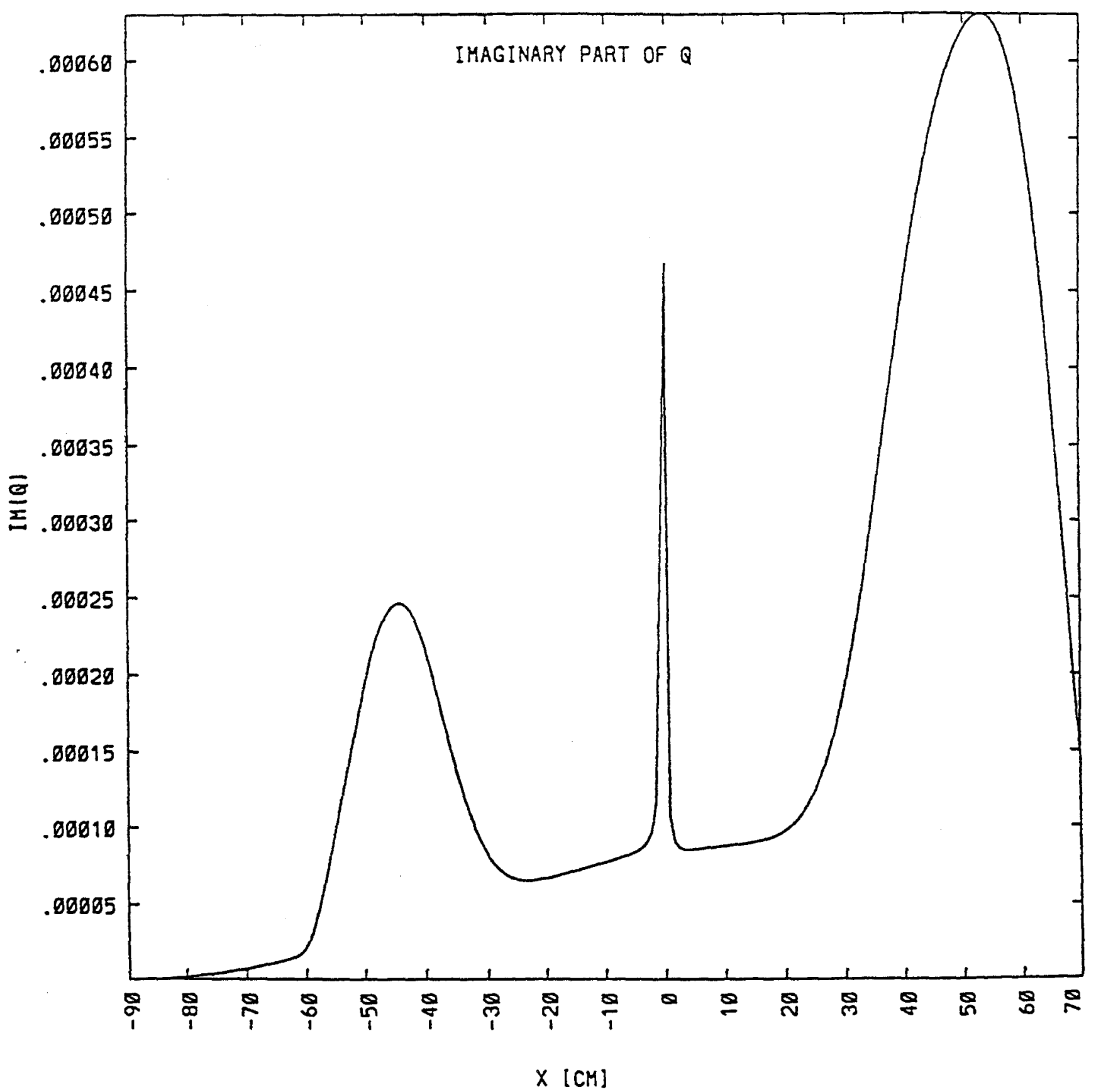

Figure 16 The imaginary part of the fast wave potential plotted as a function of the spatial co-ordinate. The parameters are the same as Fig 13 with $k_{\|}=6.6 \mathrm{~m}^{-1}$. 


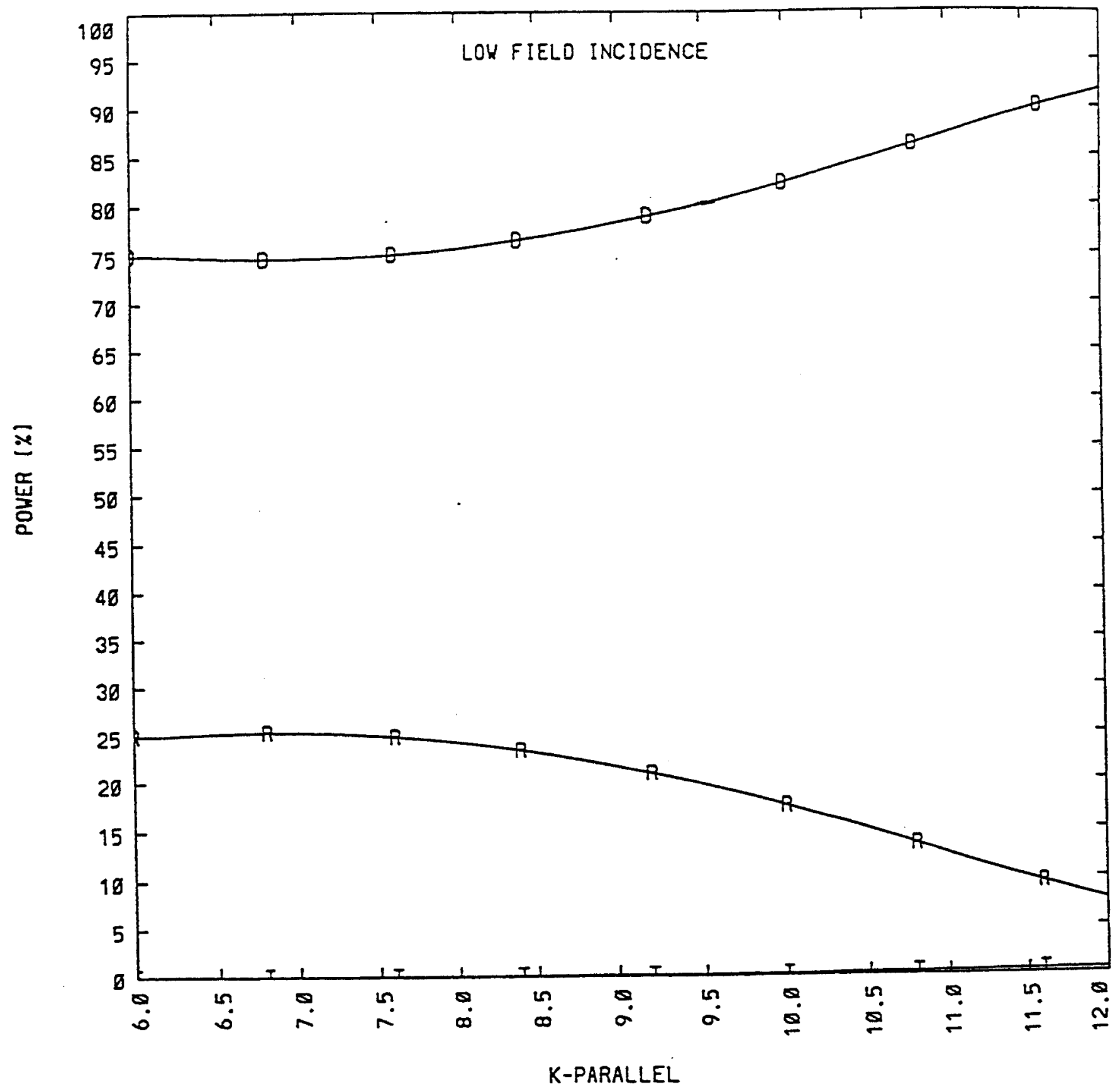

Figure 17 Transmission $(T)$, reflection $(R)$ and total absorption fractions plotted as a function of the parallel wavenumber for TFTR projected parameters: $n_{e}=$ $2 \times 10^{20} \mathrm{~m}^{-3}, B_{o}=4.8$ tesla, $R=2.62 \mathrm{~m}, T_{e}=30 \mathrm{keV}$ for a deuterium (51\%) tritium (49\%) plasma with $T_{D}=T_{T}=20 \mathrm{keV}$. 


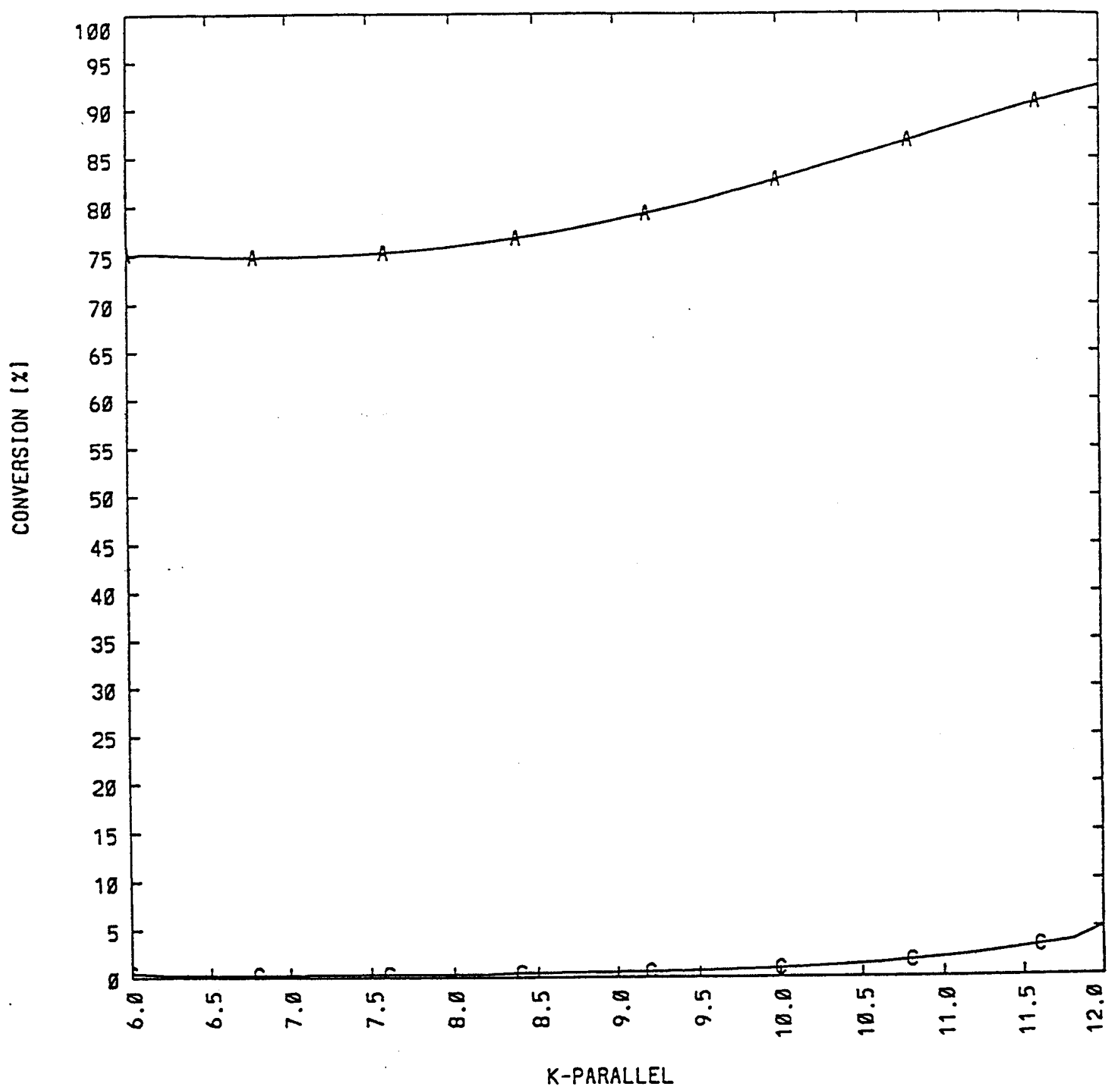

Figure 18 Comparison of total absorption (A) with the mode converted power (C) plotted as a function of the parallel wave number. The parameters are the same as Fig 17. 


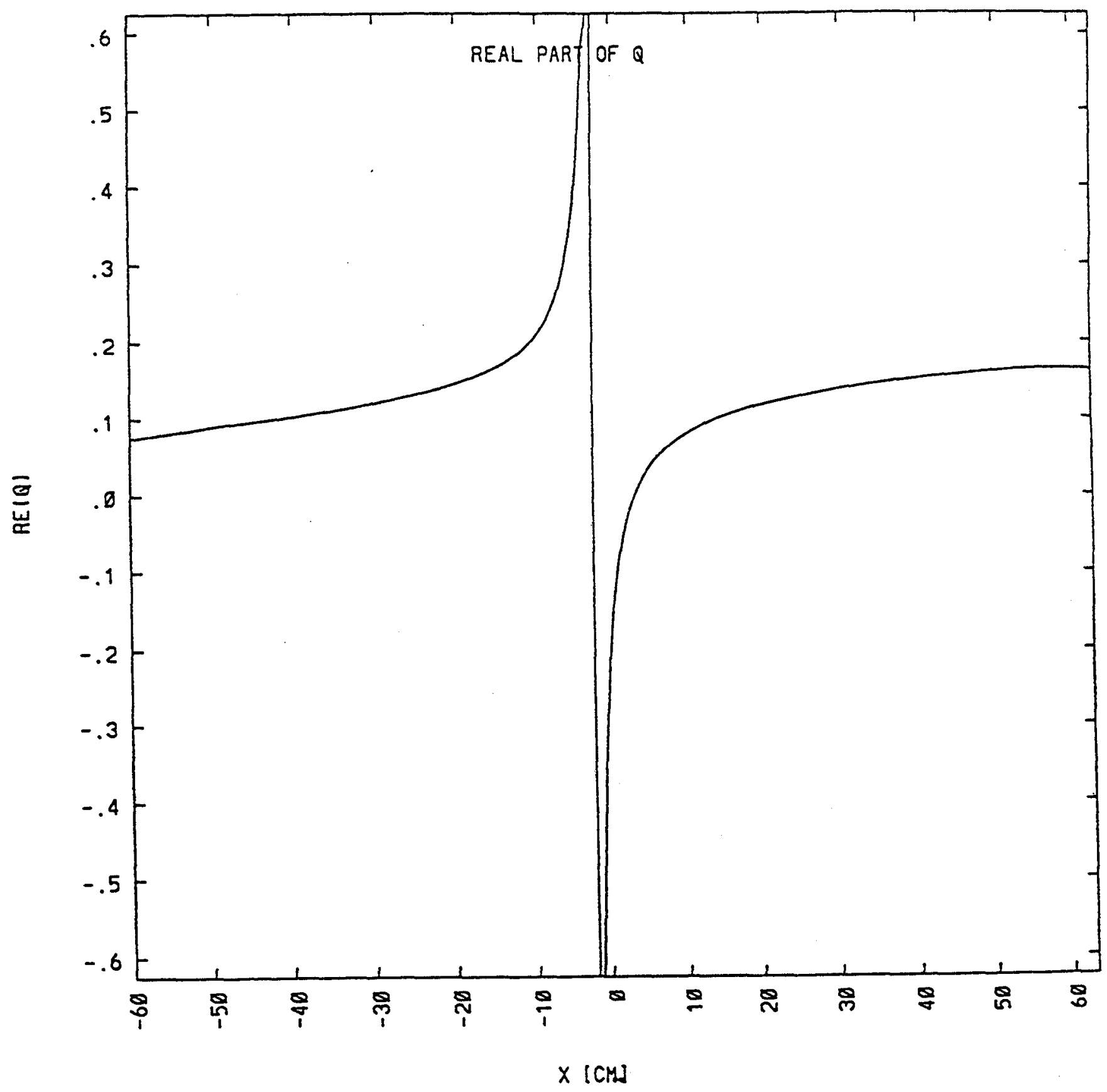

Figure 19 The real part of the fast wave potential plotted as a function of the spatial co-ordinate. The parameters are the same as Fig 17 with $k_{\|}=6.6 \mathrm{~m}^{-1}$. 


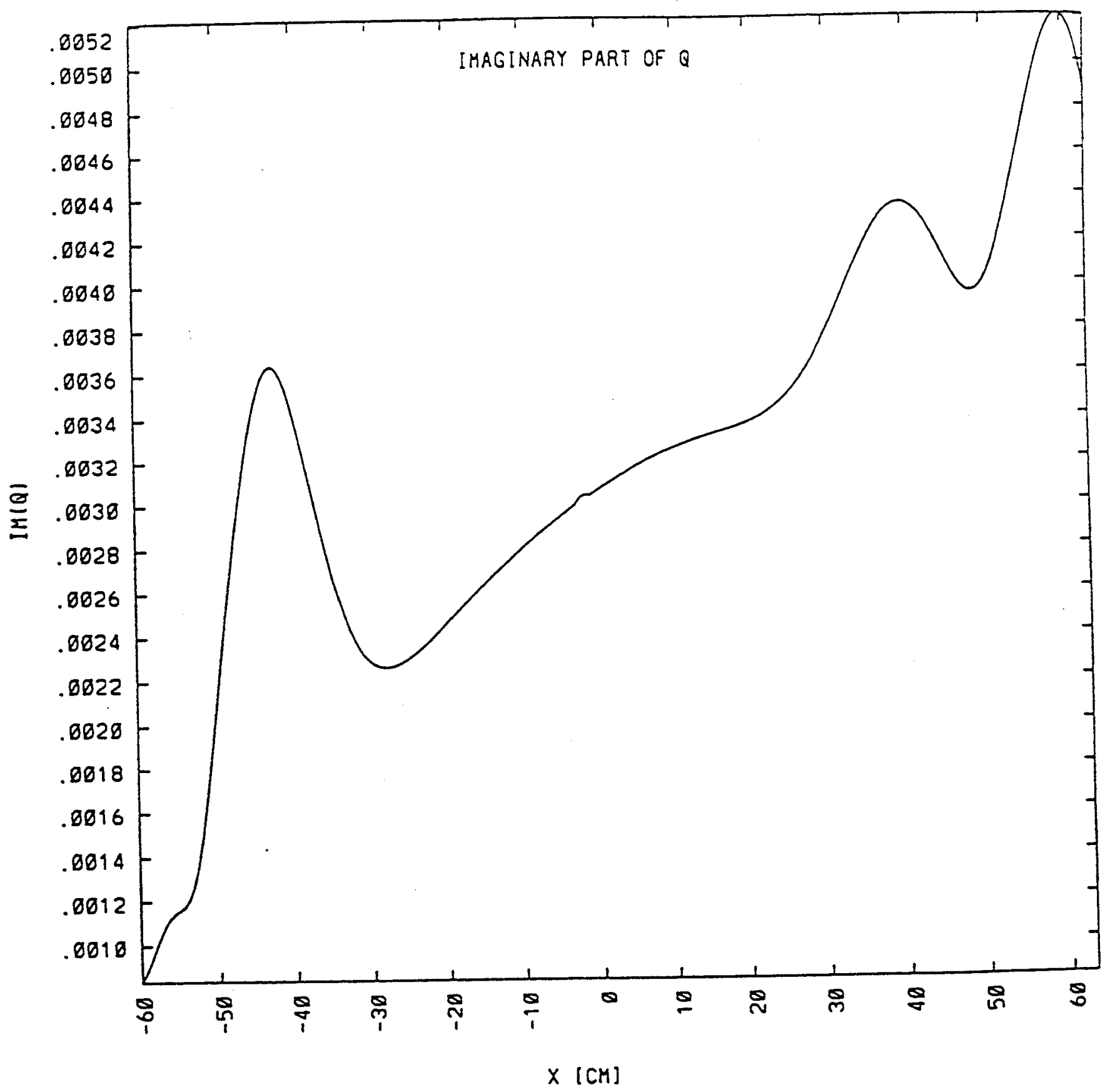

Figure 20 The imaginary part of the fast wave potential plotted as a function of the spatial co-ordinate. The parameters are the same as Fig 17 with $k_{\|}=6.6 \mathrm{~m}^{-1}$. 